\title{
CONFLICT RESOLUTION AND TRAFFIC COMPLEXITY OF MULTIPLE INTERSECTING FLOWS OF AIRCRAFT
}

by

Kyle Treleaven

B.S. in Electrical Engineering, University of Pittsburgh, 2006

Submitted to the Graduate Faculty of the School of Engineering in partial fulfillment of the requirements for the degree of M.S. in Electrical Engineering

University of Pittsburgh 2007 


\title{
UNIVERSITY OF PITTSBURGH \\ SCHOOL OF ENGINEERING
}

\author{
This thesis was presented \\ by
}

Kyle Treleaven

It was defended on

November 28, 2007

and approved by

Z.-H. Mao, Ph. D., Assistant Professor, Electrical and Computer Engineering Department

J. R. Boston, Ph. D., Professor, Electrical and Computer Engineering Department P. Loughlin, Ph. D., Professor, Electrical and Computer Engineering Department Thesis Advisor: Z.-H. Mao, Ph. D., Assistant Professor, Electrical and Computer Engineering Department 


\title{
ABSTRACT \\ CONFLICT RESOLUTION AND TRAFFIC COMPLEXITY OF MULTIPLE INTERSECTING FLOWS OF AIRCRAFT
}

\author{
Kyle Treleaven, M.S.
}

University of Pittsburgh, 2007

This paper proposes a general framework to study conflict resolution for multiple intersecting flows of aircraft in a planar airspace. The conflict resolution problem is decomposed into a sequence of sub-problems each involving only two intersecting flows of aircraft. The strategy for achieving the decomposition is to displace the aircraft flows so that they intersect in pairs, instead of all at once, and so that the resulting conflict zones have no overlap. A conflict zone is defined as a circular area centered at the intersection of a pair of flows which allows aircraft approaching the intersection to resolve conflict completely within the conflict zone, without straying outside. An optimization problem is then formulated to displace the aircraft flows in a way that keeps airspace demand as low as possible. Although this optimization problem is difficult to solve in general due to its non-convex nature, a closedform solution can be obtained for three intersecting flows. The metric used for the airspace demand is the radius of the smallest circular region (control space) encompassing all of the non-overlapping conflict zones. This radius can also be used as an indication of traffic complexity for multiple intersecting flows of aircraft. It is shown that the order of growth of the demand for control-space radius is $\Theta\left(n^{4}\right)$ for symmetric configurations of $n$ flows of aircraft. 


\section{TABLE OF CONTENTS}

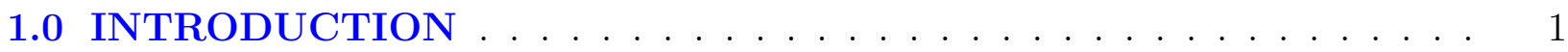

1.1 AIR TRAFFIC MANAGEMENT SYSTEM . . . . . . . . . . . . . . . 1

1.2 ISSUES OF AIRSPACE COMPLEXITY . . . . . . . . . . . . . . . 2

1.2.1 Intrinsic vs. Cognitive Complexity . . . . . . . . . . . . . . . 2

1.2.2 Complexity in a Hierarchy . . . . . . . . . . . . . . . . . 3

1.3 RELATED TOPICS . . . . . . . . . . . . . . . . . . 4

1.3.1 "Free Flight" . . . . . . . . . . . . . . . . . . 4

1.3.2 Networks of Mobile Robotic Vehicles . . . . . . . . . . . 5

1.4 COMPLEXITY-CAPACITY: A TRADE-OFF . . . . . . . . . . . 6

1.5 STRATEGIES FOR COMPLEXITY MitiGATION . . . . . . . . . . . . 7

1.6 STATEMENT OF CONTRIBUTION . . . . . . . . . . . . . . . 9

1.7 ORGANIZATION OF THE THESIS . . . . . . . . . . . . . . . 10

2.0 AIRSPACE, AIR TRAFFIC, CONFLICT RESOLUTION, AND TRAFFIC COMPLEXITY . . . . . . . . . . . . . . . . . . . 11

2.1 GENERAL CONSIDERATIONS . . . . . . . . . . . . . . . 11

2.2 MATHEMATICAL DESCRIPTIONS . . . . . . . . . . . . . . . 14

3.0 EXTENDING PREVIOUS ANALYSIS OF TWO INTERSECTING

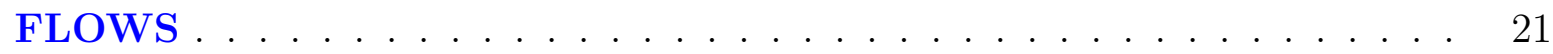

4.0 THREE INTERSECTING FLOWS OF AIRCRAFT . . . . . . . . . . 24

4.1 DETERMINING A MINIMIZING FLOW CONFIGURATION . . . . . . . 25

4.2 ALIGNing A CIRCULAR REGION TO A MINIMAL CONFIGURATION 30 4.2.1 Minimal Configuration Touches Two Interior Circles . . . . . . . . . 30 
4.2.2 Minimal Configuration Touches Three Interior Circles . . . . . . . . 34

5.0 CONSIDERATIONS FOR MULTIPLE FLOWS OF AIRCRAFT . . . 36

5.1 GENERAL OPTIMIZATION PROBLEM . . . . . . . . . . . 36

5.2 HOW TRAFFIC COMPLEXITY IS AFFECTED BY NUMBER OF FLOWS 39

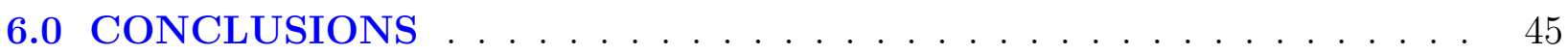

APPENDIX. PROOF OF PROPOSITION . . . . . . . . . . . . . 47

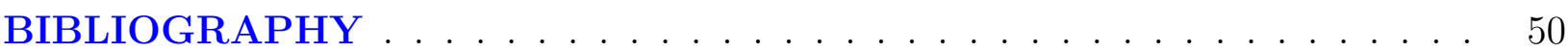




\section{LIST OF TABLES}

1 Main notation used in the paper. . . . . . . . . . . . . . . . 15 


\section{LIST OF FIGURES}

1 A general complexity hierarchy. . . . . . . . . . . . . . . . . 6

2 Conflict resolution for multiple intersecting flows of aircraft. . . . . . . . . . 12

3 A flow of air traffic. . . . . . . . . . . . . . . . . 18

4 Two-flow control strategy . . . . . . . . . . . . . . . . . . 22

5 Two cases of a three-flow triangular flow configuration. . . . . . . . . . . . . . 27

6 Determining re-parameterization of a flow given a new reference point. . . . . 29

7 Determining the slack allowed for a third circle. . . . . . . . . . . . . . . 32

8 Apollonius' circle, the encompassing circle tangent to all three inner circles. . 33

9 Multi-flow shell and band definitions. . . . . . . . . . . . . . . . . . . 39

10 The string of pearls constraint. . . . . . . . . . . . . . . . . 42

11 Encompassing circle tangent to two inner circles. . . . . . . . . . . . . . . . . 49 


\subsection{INTRODUCTION}

\section{$1.1 \quad$ AIR TRAFFIC MANAGEMENT SYSTEM}

In the United States, the Air Traffic Management (ATM) system provides the services necessary to ensure safe, orderly and efficient aircraft operations [1]. The domain of this ATM system includes the airspace over the continental United States, large portions of the Pacific and Atlantic oceans, and the Gulf of Mexico. The ATM system consists of two primary components, namely the Air Traffic Control (ATC) and Traffic Flow Management (TFM) functions. The first of these, the ATC function, ensures separation of aircraft as a means to avoid aircraft conflict or collision. The second, the TFM function, organizes aircraft into flow patterns to ensure their smooth and efficient movement. Naturally, the airspace above the United States is quite large. Therefore, in order to support the ATC and TFM functions, the airspace is partitioned among a number of Air Route Traffic Control Centers (ARTCCs). Within a Center, an airspace is further segmented, for example, into strata by altitude ("low," "high" and "super-high"), with each stratum being partitioned again into horizontal Sectors. In this way, a typical ARTCC is subdivided in 20 to 50 Sectors, which form the basic control unit within the ATM system. Sectors are designed such that the controllers assigned to a sector are able to handle the usual flow of air traffic. However, in times of increased demand or re-routing - required, for example, due to weather conditions"proven" TFM techniques such as staff reallocation and alternative airspace configurations can be used to maintain the workload of the controllers so that air traffic safety and flow efficiency remain uncompromised. The current ATM system, as describe, has a limit to the amount of air traffic it can support. 
Unfortunately, the current ATM system faces a large demand for air transportationarguably approaching its capacity. In 2006, for example, the number of passenger enplanements in the United States was nearly 658 million, and that of aircraft departures was about 10.5 million [2]. Naturally, these demands for air transportation have produced a general increase in ATC workload, but more importantly, they result in an increase in the frequency of airborne delays. Such an increase is evidence that the demand for air transportation is approaching the capacity of the current system to support it. Furthermore, these numbers are predicted to continue growing at a rapid pace in the future. According to this expected growth - and considering an increasing desire for more efficient routing - higher demand will be placed on the ATM system, which the current system may not be able to satisfy.

\subsection{ISSUES OF AIRSPACE COMPLEXITY}

This growth in demand will require a more comprehensive understanding of the relationship between the many factors of air traffic control, including: 1) air traffic patterns and control procedures, 2) environmental factors (e.g. weather-based obstacles) and airspace partitioning, and 3) the cognitive and/or computational capabilities of air traffic controllers. The various many-faceted relations between these factors have received widespread attention in the literature, and are ubiquitously referred to under the far-reaching term, "Complexity".

\subsubsection{Intrinsic vs. Cognitive Complexity}

There has been a wide range of research efforts examining the complexity of air traffic. These studies can be put very roughly into two categories: One is related to cognitive complexity, which serves as an indication of the mental workload of controllers in response to the air traffic (e.g. $[3,4,5,6]$ ); and the other is related to intrinsic complexity, which is related to metrics characterizing the airspace and traffic of aircraft or other mobile vehicles from a systems perspective (e.g. [7, 8, 1, 9, 10, 11]). 


\subsubsection{Complexity in a Hierarchy}

Despite the usual categorization of complexity into "cognitive" and "intrinsic" levels, it is sometimes treated on a spectrum. For example, Cummings stacks levels of complexity [5], claiming the "lowest" to be cognitive complexity, followed by display complexity, then organizational and finally environmental complexity. The levels of complexity are addressed as follows: Factors of environmental complexity include, for example: number of aircraft, weather, congestion, etc. These correspond to the usual elements of intrinsic complexity, and represent - from a systems perspective - "intrinsic" or inherent constraints of the system. Goals, policies and procedures are organizational elements, which in general exist to mitigate environmental complexity. However, the fact of the matter is that organizational elements usually impose further constraints on the air traffic system, which, under high demand situations, may actually add to complexity. Next addressed is display complexity. In order to facilitate human decision processes, information about a traffic situation must be communicated to human air traffic controllers as accurately as possible. In Cummings' model, display complexity is related to the possible increase in complexity from information reporting methods. Finally, cognitive complexity is the complexity associated with human controllers, and is generally assumed to correlate with the cognitive difficulty of controlling the air traffic situation — as presented, or contributed to by "higher" sources of complexityby the human controller under normal and abnormal conditions. It should be noted that Cummings' hierarchy is opposite to the order of the imposition of situational constraints. That is, environmental complexity represents the complexity arising from intrinsic system constraints - these are largely uncontrollable. Organizational constraints are imposed on top of these, arising from policies and procedures. Inefficient information displays can introduce additional constraints to cognitive complexity. Finally, the cognitive capabilities of the individual controller represents a layer of traffic control system constraints from the perspective of the ATM system. In general, a better understanding of all of the factors that impact Cognitive Complexity is important for advancing both safety and efficiency in the ATC system. 


\subsection{RELATED TOPICS}

At this point in the Introduction of this paper it is valuable to make a few digressions into the application of this work to the evolution of Free Flight and to robotics and networks of robotic agents.

\subsection{1 "Free Flight"}

Technologies in the areas of tracking, prediction and communications constantly are being developed, and increasingly can provide equipment for better navigation, communications and computation on board airborne vehicles. These technologies have made it possible to conceive of new concepts of air traffic systems, such as Free Flight, which are motivated by the desire for more efficient handling of airborne traffic. More specifically, as noted by Paielli and Erzberger: "The economics and efficiency of air transportation in the continental U.S. could be improved significantly if the rigid routing restrictions currently imposed by the Federal Aviation Administration (FAA) were relaxed to allow more direct or wind-optimal trajectories" [12]. That is, while current routing restrictions are in place to maintain safe and orderly flow of air traffic, new technological considerations could maintain or improve safety and efficiency of air traffic if those restrictions were relaxed or reformulated. Free Flight, the ultimate evolving goal of this approach was designed to allow free routing. The idea was to place the power of traffic optimization into the hands of the airlines [13], where it was thought to receive more economical, and therefore more efficient consideration than by a global controller. Free Flight calls for the dismantling of much of the current structure of the ATM system in favor of a more chaotic but more efficient management system. In that respect, Free Flight also motivates an interest in distributed traffic control, where the role of the ground-based air traffic controller is limited. Instead, emphasis is placed on control decisions based on information available locally to potential sites of air traffic conflict. This concept becomes realizable as equipment in the air approaches the reliability of ground-based equipment. 
Because of the limited availability of information and the emphasis on distributed control, there is also emphasis, to some extent, on the possibility of decentralized control strategies.

To that end, the development and subsequent implementation of any new ATM concept of operations requires one to study extensively the mechanisms by which airplanes are able to maintain separation from other airplanes at any time and in a predictable fashion.

\subsubsection{Networks of Mobile Robotic Vehicles}

It is in the emphasis for some combination of automated, distributed and decentralized decision-making mechanisms that the study of air traffic control intersects with robotics and the control of systems of multiple mobile robotic agents in a common area.

As noted by Sharma et al. [11], with the progress of technology in mobile robotics, unmanned, autonomously controlled vehicles - in a variety of environments, including airspacewill increasingly share common resources with human-controlled vehicles in the same environment. As a result, there is a focus on the development of safe and effective algorithms for autonomous decision making in large networks of mobile vehicles and similar robotic agents.

In the automation domain, cognitive complexity is superseded by the concept of "computational complexity", but the fundamental issues remain the same. That is, from a computation perspective, the term "cognitive complexity" represents only one member of an overarching class called "computational complexity" - the member concerned with humanin-the-loop control systems. Computational complexity is instead correlated with the computational difficulty of controlling a traffic situation - or any control situation - by a general controller (machine, human or machine/human).

Researchers believe that the ability of human controllers to reduce traffic congestion - for example, as air traffic density increases - and to ensure efficient and safe operation of these spaces is approaching its limits. Therefore, there are clear advantages to considering the automation of traffic control. The clearest advantage of considering automation-and the more general factors of computational complexity - include the tremendous wealth of knowledge in the theory of computing and computer science, as well as the speed, capabilities and large-scale available of modern computing systems. On the other hand, the undeniable 


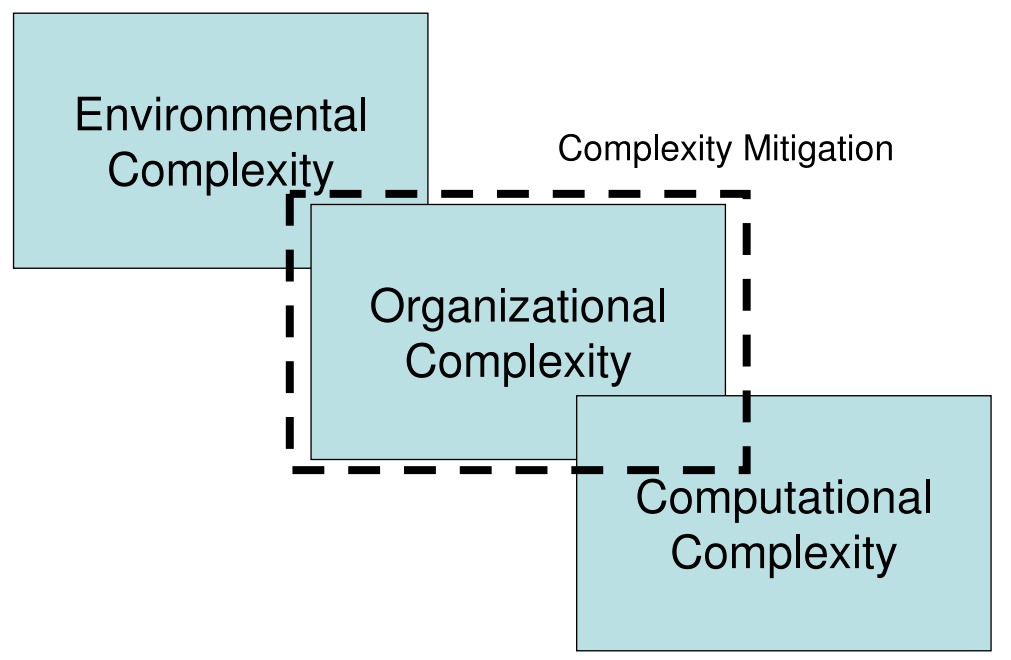

Figure 1: A general complexity hierarchy.

advantage of human-controlled or human-in-the-loop systems lies in the robustness of cognitive capabilities to handle unexpected outcomes and to make important decisions where problems may be poorly-defined or beyond the scope of the computer system to anticipate or evaluate. A major related consideration in control systems - especially in areas like air traffic and other wide-scale public systems control - is an issue of public trust of autonomous systems. Ideally - and currently - a hybrid approach utilizing computer-augmented human cognitive functions is implemented.

\subsection{COMPLEXITY-CAPACITY: A TRADE-OFF}

The human-computer relation described above recalls the layered complexity of Cummings, and specifically the "display" complexity stratum as the bridge between organizational and cognitive complexities. Therefore, in the pattern of Cummings, let us consider a new complexity hierarchy (see Figure 1) for a generalized system, consisting of 1) environmental complexity, 2) organizational complexity, and 3) computational complexity, where computational complexity supersedes factors of display and cognitive complexity. It can be argued 
that the computational complexity and the capacity of an air traffic system exhibit a complicated trade-off behavior. Let us consider the concept of "organizational capacity", as distinguish from "computational" (realizable) capacity. The respective nomenclature is derived from the lowest level of complexity considered in their respective definitions. We define organizational capacity to represent a measure of the capacity of a system without considering practical bounds on computational availability. That is, this measure assumes that for any system operating subject to its organizational capacity - given both environmental and organizational complexity factors - the means are theoretically available to provide the computation required to support the system, e.g. aircraft flow. Loosely speaking, this implies that reasonable control strategies exist in the decision space, and therefore it is possible to construct a machine to find them. The computational complexity and capacity measures then are related to the density and searchability of reasonable strategies in the decision space, or the difficulty associated with finding one. Naturally, all real systems have practical limitations to their computational capabilities, so computational capacity may not reach organizational levels. Such computational limitations are exemplified by the observed interaction of cognitive complexity factors with the current ATM system, wherein the realizable capacity must be limited in order to maintain safe and effective air traffic control. That is, a more effective computational entity could, in theory, support a higher capacity under the current ATM system, but human controllers cannot.

\subsection{STRATEGIES FOR COMPLEXITY MITIGATION}

It is valuable now to consider the concept of structural abstraction, as suggested by Histon [3], which can be categorized as an element of organizational complexity under Cummings' hierarchy - and now ours. The issue of abstraction raised by Histon addresses a means of representing the essential characteristics of a system model in a more cognitively compact form that is manageable within the constraints of human memory and processing limitations. We can easily extend this definition to address a more general computing entity as well. The most common example of structural abstraction in air traffic control is the organization of 
aircraft into air traffic flows. Histon proposes that structural abstractions, such as air traffic patterns, can limit the computational complexity of a system in order to support larger capacities under regular traffic conditions. However, structural abstractions may represent further constraints on possible system states and thus strike a compromise between computational complexity and organizational capacity. It is observed, in general, that increasing computational capacity involves mitigating computational complexity. If this mitigation is achieved by organizational factors, then it often implies a decrease in organizational capacity. In turn, the organizational capacity of a system forms an upper bound on the computational capacity, by merit of its situation further down the complexity hierarchy. The goal then of the system designer is to simultaneously maximize computational capacity - usually through complexity reduction - while maintaining a controllable (and hopefully minimal) descent in the organizational capacity from environmental or intrinsic levels. In this work, we specifically focus on the extent to which types (or one type) of structural abstractions strike a reasonable compromise between computational complexity and organizational capacity. As suggested by Histon et al. [3], the traffic complexity and workload of ATC controllers can be reduced by reorganizing the air traffic into standard flows or other structured traffic patterns. Following this idea, we concentrate on the traffic pattern of aircraft flows.

Characteristics and conflict resolution of aircraft flows have been investigated in several studies. For example, non-overlapping or non-intersecting flows of aircraft have been studied thoroughly in [14, 15, 16]. Mitchell et al. [14] and Krozel et al. [15] have investigated the problem of estimating the capacity of an airspace region during weather events. In both studies, they considered a model where the aircraft routes pass through the airspace with constant-width air lanes. These lanes are not allowed to overlap among each other. Bayen et al. [16] have developed a partial differential equation to describe the dynamics of traffic along an aircraft route or airway, and further generalized their analysis to a network of interconnected airways. Based on their network model, they investigated the detailed characteristics of unidirectional, non-intersecting air traffic. For intersecting aircraft flows, Mao, Feron et al. [17, 18, 19, 20] have conducted research investigating the stability of the aircraft flows under both decentralized and centralized conflict-avoidance rules. They considered two and three intersecting flows of aircraft, respectively, but did not continue the 
analysis for more than three intersecting flows. The work of Mao et al. includes models where conflict resolution maneuvers are modeled as instantaneous deviations, as well as the more realistic vision of resolution by heading change maneuvers. Their capacity-maximizing proof of system stability for two intersecting air traffic flows asserts their model as a structural abstraction demanding further review. An abstraction of this type provides a hard ceiling on capacity in order to guarantee a hard bound on computational complexity. This type of capacity-complexity relationship lends itself well to automated control, where a high degree of "certainty" of stability is desirable. Meanwhile, the model itself is shown to be applicable in both centralized and decentralized control strategies. It might be argued that the dynamic computational nature of the current ground-based and human cognitive implementation of air traffic control would achieve optimal capacity when considering a more fluid capacitycomplexity relationship. However, as noted, the eventual maturation of Free Flight, as well as applications in the networking of mobile robotic or automatically controlled agents, may benefit from this higher degree of stability.

\subsection{STATEMENT OF CONTRIBUTION}

In this study, we follow upon the research efforts by Mao, Feron et al. [17, 18, 19, 20], and propose a general framework for the conflict resolution of multiple intersecting flows of aircraft. As pointed out by [20], the study for more than three intersecting flows tends to be very complicated if we accept as a constraint that each aircraft is allowed to make only one maneuver for conflict resolution. However, if the constraint is relaxed to allow multiple, sequential maneuvers for individual aircraft to avoid conflicts, the analysis becomes simpler: The conflict resolution problem can be decomposed into a sequence of sub-problems each involving only two intersecting flows of mobile agents. Then, by using the obtained analytical results for two intersecting flows [17], we can estimate the performance bounds as well as safe space capacity for more than three intersecting flows. Consideration of the general traffic scenario of multiple intersecting flows of aircraft stresses some of the more difficult problems arising in conflict management, including the "domino effect." Based on investi- 
gation of traffic complexity for such traffic scenarios, this paper aims to obtain insight into deeper problems like estimation of air space capacity, prediction of traffic congestion, computation of performance bounds for optimization algorithms under various conflict resolution architectures, and even evaluation of cognitive workload of human controllers.

\subsection{ORGANIZATION OF THE THESIS}

The rest of this paper is organized as follows. Chapter 2 describes the models for airspace, air traffic flows, aircraft maneuvers, conflict resolution, and traffic complexity. This section also introduces a general framework for conflict resolution of multiple intersecting flows of aircraft based on decomposing the original problem into a sequence of two-flow problems. Chapter 3 considers the conflict resolution within a conflict zone, which involves only two intersecting flows of aircraft. Using the analytical results obtained from two intersecting flows, Chapter 4 investigates the scenario of three intersecting flows and develops an algorithmic procedure to determine the most compact conflict-free configuration for the three flows. Chapter 5 studies the increasing complexity of the multiple flow problem with numbers of aircraft flows larger than three. The last Chapter is the conclusion. 


\subsection{AIRSPACE, AIR TRAFFIC, CONFLICT RESOLUTION, AND TRAFFIC COMPLEXITY}

In this section we will outline and describe the models used to analyze the complexity of air traffic in a planar airspace and to determine measures of conflict resolution. We develop models for the controlled airspace and for the arrangement of aircraft into traffic flows as proposed in previous work. Finally, we examine the degree to which we may exercise control over the system variables in order to guarantee conflict-free transportation within a controlled airspace.

\subsection{GENERAL CONSIDERATIONS}

Following the work of Mao, Feron, et al. [17, 19, 20], we consider only a planar, or twodimensional, ATC space, or control space. For simplicity of analysis, the control space examined in this paper is a circular region, and is parameterized by a radius, $R$ (see Figure 2). In this model, we assume that aircraft travel at the same altitude and with the same speed, and use only horizontal maneuvers for conflict resolution. Potential conflicts occur at flow intersections, which are inherent to non-parallel flows. Conflict avoidance near these points requires a maneuver, which, as in the model of [17], consists of lateral "stray" and "return" deviations of individual aircraft about the flow line.

In the vicinity of the control space, we consider air traffic to be arranged into traffic flows. Aircraft within a flow have uniform velocity along the flow direction and may be packed to arbitrary density subject to the requirements of individual aircraft separation. As in previous work, the amount of required separation $D_{\text {sep }}$ between aircraft is established by 


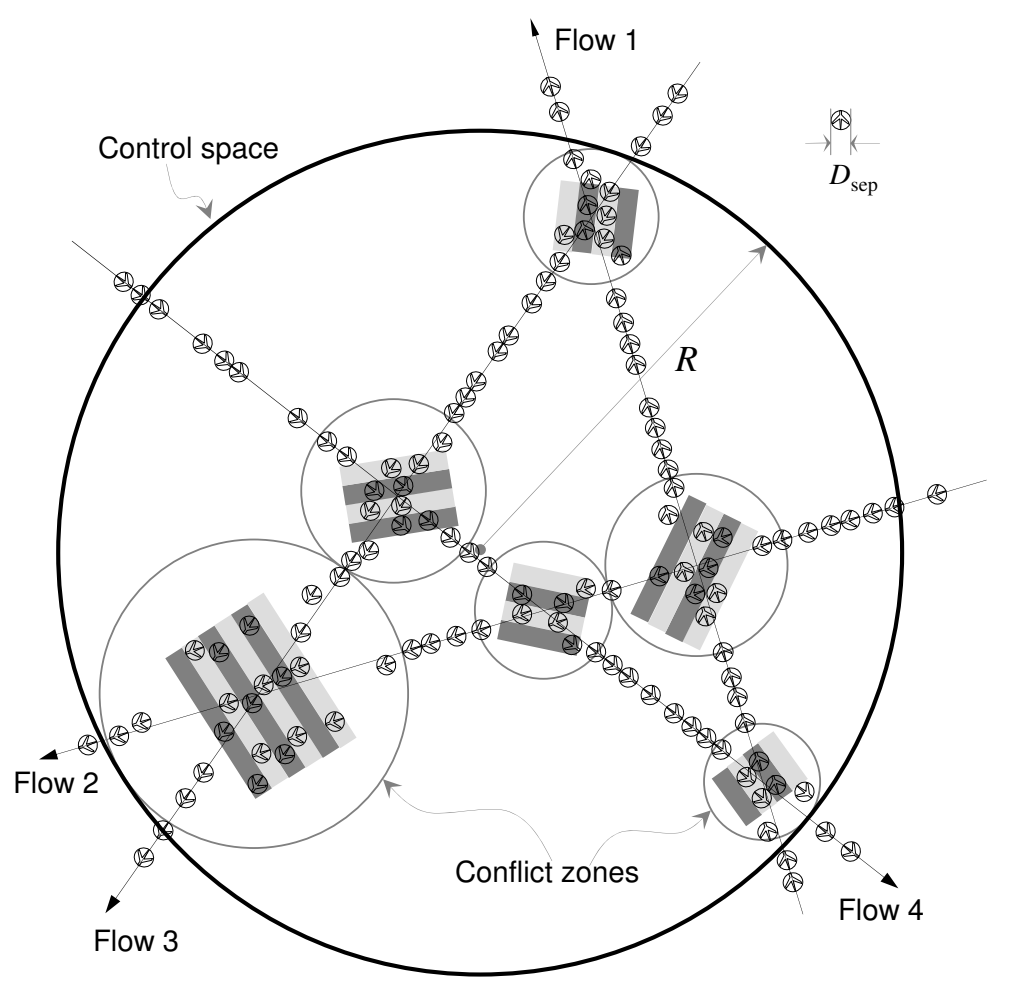

Figure 2: Conflict resolution for multiple intersecting flows of aircraft. Our strategy is to displace flows so that they intersect in pairs and the resulting conflict zones have no overlap. A conflict zone surrounding the intersection of a pair of aircraft flows is defined as an area demanded to guarantee that aircraft approaching the point of conflict can avoid such conflict completely, without straying outside of the area. Within each conflict zone, aircraft may resolve conflict using either a decentralized strategy [17] or a centralized strategy [20] (shown in the figure is the centralized strategy, which is based on space partition-light and dark "aisles" are alternatively arranged to partition the airspace - see [20] for details). 
a circular buffer of radius $D_{\text {sep }} / 2$ surrounding individual aircraft. The Federal Aviation Administration separation distance is 5 nautical miles $(\mathrm{nm})$. This circular buffer, then, is known as the aircraft protected zone, and the fundamental criterion for conflict resolution is that protected zones between aircraft should not overlap. A traffic flow, by the definition used up to this point, supports arbitrary aircraft density along the direction of flow, but is precisely one aircraft separation in width. While for the present time we continue to consider a flow as being one aircraft wide, it may be useful in the future to consider flows allowing multiple aircraft widths instead.

At this point we assume no parallel or anti-parallel flows, such that all flow pairs have a single intersection in the finite plane. Parallel flows can be handled fairly easily after developing an intermediate algorithm without considering them at first. For each flow out of $n$ flows there are $n-1$ other flows with which it might intersect. A number of mutually nonparallel flows $n$ converging on a control space implies $n-1$ points of potential conflict along a single flow line, or $n(n-1) / 2$ intersections in total (the number of pairwise combinations of flows).

In our model we consider an area, called the conflict zone, surrounding the intersection, or point of conflict (Figure 2). The conflict zone demands some additional area, apart from the nominal area of the aircraft protected zone, to guarantee that aircraft approaching the point of conflict can avoid such conflict completely, without straying outside of the area. Outside of these zones, the aircraft paths along flows are treated as straight lines, without misbehavior.

In this paper our strategy of conflict resolution for multiple intersecting flows of aircraft is to displace the flows so that they intersect in pairs, instead of all at once, and so that the resulting conflict zones have no overlap (Figure 2). We hope to accomplish this objective in a way that keeps airspace demand as low as possible, where the airspace demand is measured as the radius of a circular region encompassing all the conflict zones. In this paper we adopt an oversimplified model of complexity, which is nevertheless adequate for our analysis. We note our assumption that complexity is inversely related, loosely, to capacity and adopt our measure accordingly: The lowest airspace demand, characterized by the radius of the smallest circle that can encompass an arrangement of conflict zones, provides a natural indication of 
the traffic complexity for multiple intersecting flows of aircraft. This measure represents an index, to some degree, of the resources that must be allocated to guarantee systems stability, which conforms to the nature of a measure of complexity. Our adopted indication of traffic complexity is a function of the number and orientation of the aircraft flows entering the control space.

Note that arranging flows in the above manner allows us to apply the decentralized ATC algorithm and simple stability conditions of [17] on a per conflict zone basis. Therefore, we regard the conflict zone as the fundamental stable unit in our model. Here, the definition of stability follows the one proposed in [17]: The intersecting aircraft flows are defined to be stable if all conflicts are resolved and the deviation of each aircraft trajectory from its original track, due to the requirement for conflict resolution, remains bounded. In addition, the control space is stable if all conflict zones avoid overlap and are contained within the control space.

\subsection{MATHEMATICAL DESCRIPTIONS}

While the concept of the air traffic flow remains largely the same as in previous work, we will apply a slightly different flow description, relying more heavily on vector analysis. First, we establish a line describing the path of the flow. This line represents the set of all points where an aircraft (center of a protected zone) might lie. We choose the center of the circular control space as a natural reference point from which to define the location of the flow line. In the model employed in this paper, as seen in Figure 3, a flow is specified by two parameters: $\theta$, the direction of a vector normal to or "supporting" the line, and $d$, the lateral displacement along that direction of the flow from the center of the circular control space. For the convenience of the reader, these and other main notation used in this paper are summarized in Table 1. 
Table 1: Main notation used in the paper.

\begin{tabular}{|c|c|}
\hline Notation & Definition \\
\hline$b$ & Buffer coefficient in determining the radius of a conflict zone $(b>1)$ \\
\hline$d$ & $\begin{array}{l}\text { Lateral displacement of a flow along the direction of the flow from } \\
\text { the center of the circular control space }\end{array}$ \\
\hline$d_{i}$ & $\begin{array}{l}\text { Lateral displacement of flow } i \text { with respect to the center of the circular } \\
\text { control space }\end{array}$ \\
\hline$d_{\text {lower }}$ & A lower bound of $d_{\min }$ \\
\hline$d_{\min }$ & $\begin{array}{l}\text { Smallest } d \text { that ensures non-overlapping of any conflict zones in the } \\
\text { symmetric configuration of } n \text { flows }\end{array}$ \\
\hline$d_{\text {upper }}$ & An upper bound of $d_{\min }$ \\
\hline d & $\left(d_{1}, \ldots, d_{n}\right)^{T}$ \\
\hline$D_{\text {sep }}$ & Amount of required separation between two aircraft \\
\hline$e_{i}$ & $\begin{array}{l}\text { Length of the } i \text {-th edge of the triangle formed in the three-flow sce- } \\
\text { nario }\end{array}$ \\
\hline$K$ & $\begin{array}{l}\text { Total number of shells in the symmetric configuration of } n \text { aircraft } \\
\text { flows }\end{array}$ \\
\hline$l^{(k)}$ & $\begin{array}{l}\text { Distance from the origin to a } k \text {-th shell intersection in the symmetric } \\
\text { configuration of } n \text { flows }\end{array}$ \\
\hline$n$ & Total number of aircraft flows \\
\hline$r_{i}$ & $\begin{array}{l}\text { Radius of the } i \text {-th conflict zone in the scenario of three intersecting } \\
\text { flows }\end{array}$ \\
\hline$r_{j k}$ & $\begin{array}{l}\text { Radius of the conflict zone surrounding the intersection between flows } \\
j \text { and } k\end{array}$ \\
\hline$r^{(k)}$ & $\begin{array}{l}\text { Radius of any conflict zone in the } k \text {-th shell in the symmetric config- } \\
\text { uration of } n \text { flows }\end{array}$ \\
\hline$R$ & Radius of the control space \\
\hline
\end{tabular}

continued on next page. 
Table 1 (continued).

\begin{tabular}{|c|c|}
\hline Notation & Definition \\
\hline$R_{\min }$ & $\begin{array}{l}\text { Radius of the smallest circle that can host a feasible arrangement of } \\
\text { the conflict zones }\end{array}$ \\
\hline $\mathbf{u}$ & Support directional vector of a flow \\
\hline $\mathbf{u}_{i}$ & Support directional vector of flow $i$ \\
\hline$\left(x_{\mathrm{c}}, y_{\mathrm{c}}\right)$ & Coordinate of the center of the encompassing circle \\
\hline$\left(x_{i}, y_{i}\right)$ & Coordinate of the $i$-th intersection in the scenario of three flows \\
\hline$\left(x_{j k}, y_{j k}\right)$ & Coordinate of the intersection between flows $j$ and $k$ \\
\hline $\mathbf{x}_{\mathrm{c}}$ & $\left(x_{\mathrm{c}}, y_{\mathrm{c}}\right)^{T}$ \\
\hline $\mathbf{x}_{i}$ & $\left(x_{i}, y_{i}\right)^{T}$ \\
\hline $\mathbf{x}_{j k}$ & $\left(x_{j k}, y_{j k}\right)^{T}$ \\
\hline$\alpha_{i}$ & The $i$-th inner angle of the triangle formed in the three-flow scenario \\
\hline$\theta_{i}$ & Angle of the support directional vector of flow $i$ \\
\hline$\theta$ & $\left(\theta_{1}, \ldots, \theta_{n}\right)^{T}$ \\
\hline$\lambda^{(k)}$ & $\begin{array}{l}\text { Normalized distance from the origin to a } k \text {-th shell intersection in the } \\
\text { symmetric configuration of } n \text { flows }\end{array}$ \\
\hline$\phi_{i}$ & $i$-th encounter angle between two flows in the three-flow scenario \\
\hline$\phi^{(k)}$ & $\begin{array}{l}\text { Angle between two flows intersecting in the } k \text {-th shell in the symmet- } \\
\text { ric configuration of } n \text { flows }\end{array}$ \\
\hline
\end{tabular}


Loosely speaking, these parameters describe the magnitude and direction, respectively, of a support vector. The line is understood to lie perpendicular to its support direction, as seen in Figure 3. The vector equation of the line is derived below.

We consider the support directional vector, a unit vector

$$
\mathbf{u}=\left[\begin{array}{c}
\cos \theta \\
\sin \theta
\end{array}\right]
$$

such that the support vector itself is $d \cdot \mathbf{u}$. Then a positional vector $\mathbf{x}=(x, y)^{T}$ lying on the flow line, referenced from the support of the flow, satisfies orthogonality with the direction of support: $\mathbf{u}^{T}(\mathbf{x}-d \cdot \mathbf{u})=0$. Here, the operator $(\cdot)^{T}$ indicates vector transpose. Therefore, $\mathbf{u}^{T} \mathbf{x}=d \cdot \mathbf{u}^{T} \mathbf{u}=d$, i.e.,

$$
\mathbf{u}^{T}\left[\begin{array}{l}
x \\
y
\end{array}\right]=d .
$$

Note that the line equation does not completely describe the flow. We still need to know which way the traffic is flowing along the line. To complete the description we need one more piece of information: the orientation of the flow (left or right-handed). At this point it becomes important to distinguish between the support vector $d \cdot \mathbf{u}$, and the support directional vector $\mathbf{u}$. If we let $d$ take on positive or negative values (displacements to the right or left, respectively), then we can let $\theta$ correspond uniquely to the flow direction and orientation. That is, we consider that the flow lies normal to, and is oriented anti-clockwise from, its support directional vector $\mathbf{u}$. The support vector is then simply regarded as the position on the flow line which is closest to the control space origin.

We have not yet selected a reference from which to define the direction $\theta$. However, we note that rotations of a described air traffic system about the control space center have no effect on the system stability or complexity. Therefore selection of a coordinate system reference angle is largely arbitrary, and we leave it as that. Without loss of generality, flows are indexed in ascending order of the angle $\theta_{i} \in[0,2 \pi)$ for $i=1, \ldots, n$, where $n$ flows are considered. In an attempt to avoid confusion in future sections of the paper, we note that the angle $\theta$, describing the support directional vector, is a full $\pi / 2$ radians (a quarter turn) less than an angle to the flow line itself. We choose to keep this convention because the 


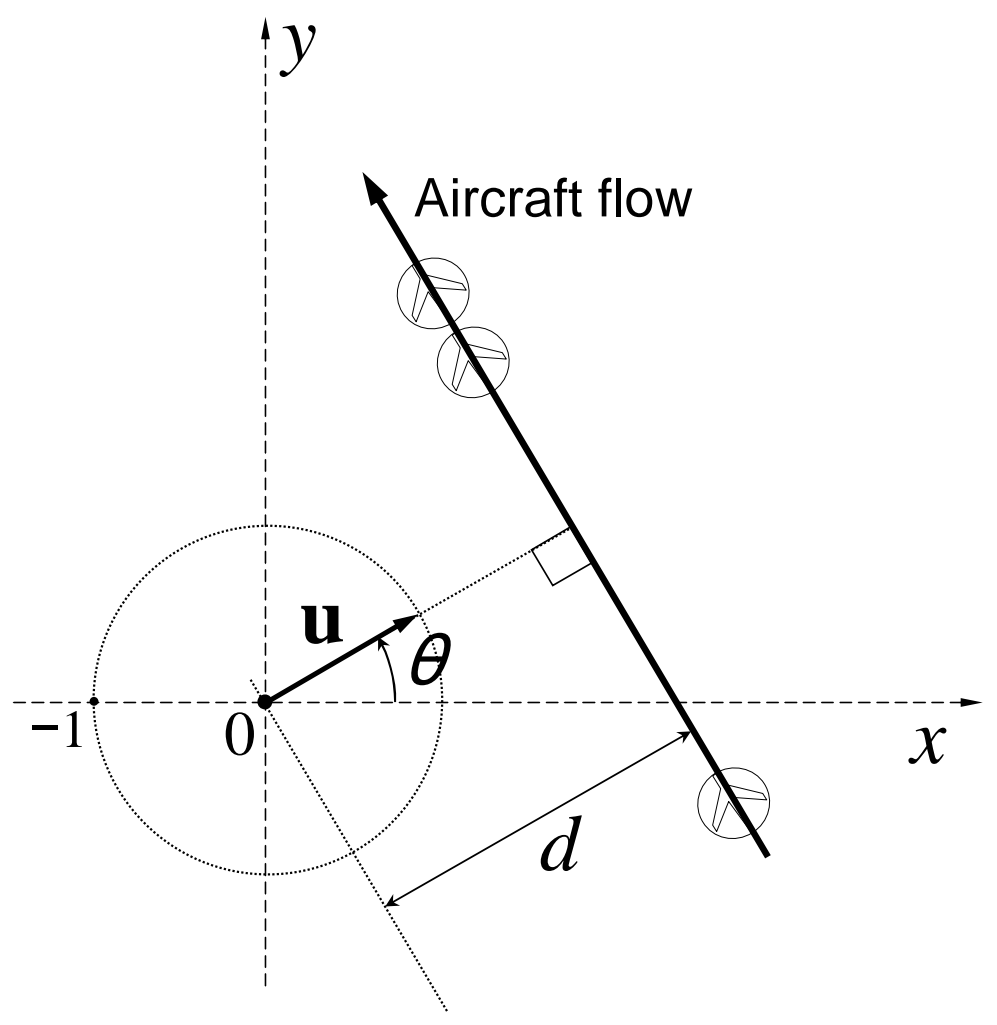

Figure 3: A flow of air traffic, defined by a support direction $\theta$ (or a support directional vector, a unit vector $\mathbf{u}$ ) and magnitude of displacement $d$. The direction of air traffic flow along the flow line is normal to and anti-clockwise from the direction of support. 
description of the flow line by a support vector simplifies the mathematics. Once again, we emphasize that a full system rotation is regarded as meaningless in terms of impact on the problem solution. It is therefore acceptable in practice, if one is more comfortable, to consider $\theta$ to be the flow direction instead of the direction of support. Mostly, one need only be cautious of these issues when the analyses are used to generate graphics.

The trajectories of flows are parameters given to the problem. That is, we are given a set of $n$ angles $\left\{\theta_{i}\right\}$, or a vector $\boldsymbol{\theta}$, defining flow orientations (support directions). However, by displacing flows laterally, we have some control over the locations of intersections. Therefore, our conflict resolution strategy in this paper is limited to the selection of a flow configuration, or a set of lateral displacements of the flows entering the control space. In the analyses of sections to follow, we will handle these displacements as a sequence $\left\{d_{i}\right\}$, or as a vector $\mathbf{d} \in \mathbb{R}^{n}$ representing the same sequence. In later sections we will develop expressions for the locations of flow intersections as a function of these displacements.

A natural default configuration is such that $d_{i}=0$ for all flows $i=1, \ldots, n$. In this configuration the flows approach the circular region normal to the boundary surface, and as such, all lines intersect each other at the center of the control space, or origin of the coordinate system. The described situation is reminiscent of the stability problem in [20], for which stability became difficult to prove with even as few as three flows. The mentioned stability problem also precludes the possibility of a decentralized algorithm for conflict avoidance for $n$ flows in general. That is, from the perspective of this work, the default configuration is not considered to be a stable configuration.

The nature of our problem is to find a configuration of flows, by way of a set of lateral displacements, such that all conflict zones are contained in the control space and none overlap. However, our primary focus in this paper is to determine the requirements placed upon the control space such that a satisfactory, or feasible, configuration of flows can be found. That is, if $\mathcal{D}_{\text {feas }}$ is the set of all feasible configurations $\mathbf{d}$ for a given control situation $\{\boldsymbol{\theta}, R\}$, then we seek to characterize the set

$$
\mathcal{R}_{\text {stable }}(\boldsymbol{\theta}) \equiv\left\{R \mid \mathcal{D}_{\text {feas }}(\boldsymbol{\theta}, R) \text { is not empty }\right\}
$$


Clearly, if we can find the smallest element of $\mathcal{R}_{\text {stable }}(\boldsymbol{\theta})$, denoted by $R_{\text {min }}$, then any $R$ greater than $R_{\text {min }}$ should also belong to $\mathcal{R}_{\text {stable }}(\boldsymbol{\theta})$. Therefore, we know that (2.3) adheres to the form

$$
\mathcal{R}_{\text {stable }}(\boldsymbol{\theta})=\left\{R \mid R \geq R_{\min }\right\}
$$

The requirements placed upon a control space then are described by a minimal control space, which is the smallest circle that can host a feasible arrangement of the conflict zones; hence the use of this metric as an indication of airspace complexity. 


\subsection{EXTENDING PREVIOUS ANALYSIS OF TWO INTERSECTING FLOWS}

In this section we review some of the background in stable conflict resolution for two flows of aircraft. In fact, the problem of the stability for a system of two intersecting flows is tantamount to the problem of stability within the conflict zone. We can use the results of $[17,20]$ to determine a stability condition for the size of a conflict zone. As discussed in Section 2.1, this conflict zone becomes the fundamental stable unit of airspace considered in this paper.

The conflict zone is an area buffer surrounding the intersection, or potential point of conflict between a pair of flows. Inside the conflict zone, conflict resolution is achieved by lateral "stray" deviations away from the flow line. For example, the aircraft in Figure 4 are arranged into "lanes," such that all aircraft in Flow 1 are situated in the light-colored lanes, while aircraft from Flow 2 are situated in the dark-colored lanes. It is argued that from the perspective of, or relative to, aircraft in Flow 1, the aircraft in Flow 2 are moving parallel to the orientation of the lanes. The aircraft in Flow 2 percieve the motion of aircraft in Flow 1 the same way. Therefore, as long as aircraft adhere to their respective lane assignments, conflict resolution is guaranteed and aircraft pass by each other in a comb-like pattern. Once these aircraft have cleared the point of conflict they may maneuver into place once again upon the flow line.

It turns out that the magnitude of a lateral deviation - the distance to the nearest safe lane - is bounded, and Mao, Feron, et al. provide a tight bound on the deviations required for the conflict-free crossing of two aircraft flows. This bound applies to both a decentralized (first-come, first-served) architecture [17] and a centralized architecture of conflict resolution, or in this case "lane-assignment [20]." The deviation bound is determined by the encounter 


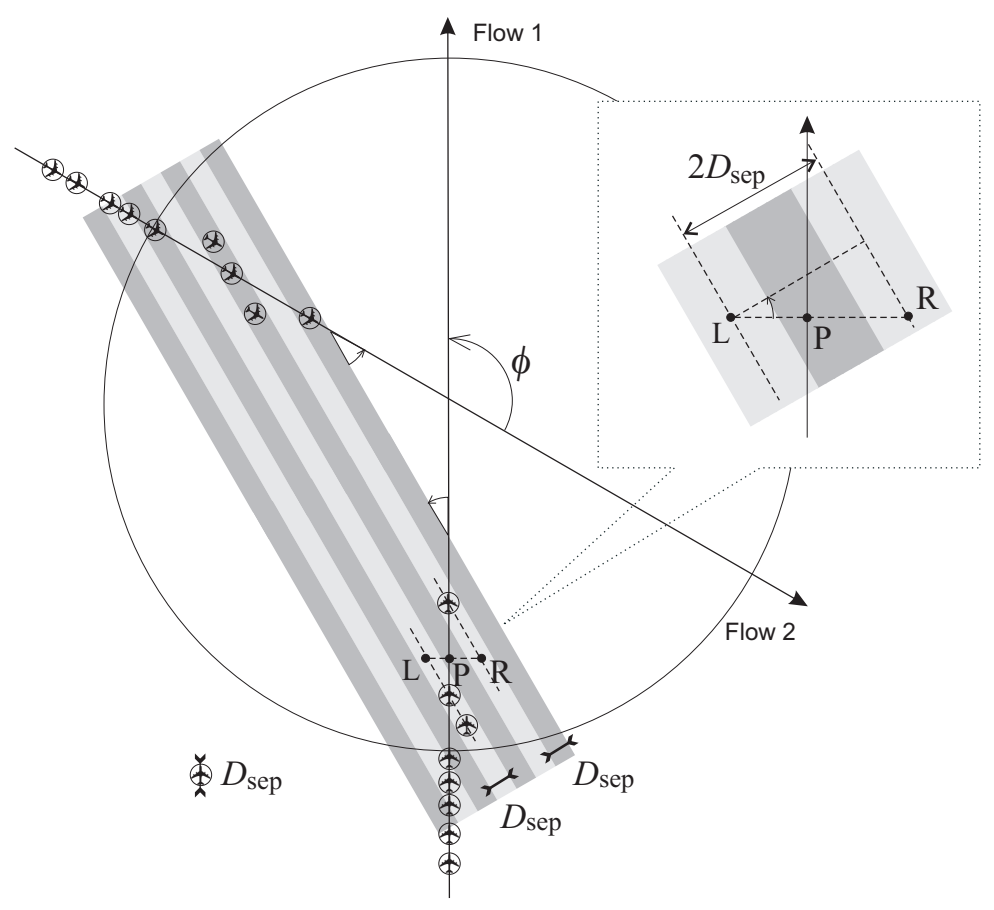

Figure 4: Two-flow control strategy; determination of control within the conflict zone.

angle $\phi$ of the two flows, as shown in Figure 4:

$$
L=\frac{D_{\text {sep }}}{\sin (\phi / 2)} \text {. }
$$

The derivation of this bound is presented in more detail in $[17,20]$. This derivation assumes that the speed of aircraft in Flow 1 is the same as the speed of aircraft in Flow 2; however, the work can be extended to unequal speeds. The important finding is that the size of the conflict zone is independent of its placement within the control space; it is independent of the selection of flow displacements.

We use the deviation bound $L$ to determine the size of the conflict zone required for stable conflict resolution of two aircraft flows. The model in $[17,20]$ considers instantaneous deviations only, while these maneuvers are not entirely realistic. Individual aircraft need some lead space in which to make a series of heading changes in order to realize such a deviation. Additionally, an actual instantaneous deviation at the surface of a circular region would place the aircraft outside of the region itself, which is contradictory to the nature of the 
conflict zone - aircraft should not stray from within. We must consider a buffer coefficient $b(L)$ in determining the radius of the conflict zone

$$
r=b(L) L
$$

where $r$ is the demanded radius of the conflict zone and $L$ is the upper bound of aircraft lateral displacement determined by (3.1). The buffer coefficient $b(L)$ is a function of $L$, but can be approximated by a constant $b$ ( $b$ should be larger than 1$)$, since the lead time for a lateral displacement is considered its dominant factor. In other words, to achieve a deviation $L$ perpendicular to the flow line, we assume the aircraft requires a long proportional lead of length $b L$. This assumption corresponds, for example, to a lateral deviation realized by a pair of complementary heading changes.

Using the constant approximation for $b(L)$ greatly simplifies the mathematical analysis. Based on this simplification together with (3.1) and (3.2), we have

$$
r=\frac{b D_{\mathrm{sep}}}{\sin (\phi / 2)}
$$

As far as calculating airspace demand for two intersecting flows is concerned, this result is immediately applicable. For two intersecting flows, the control space becomes simply the conflict zone itself, and the default configuration $\left(d_{i}=0\right.$ for both flows) realizes this minimal area. The two flow situation is in fact the only situation where the default configuration is the minimizing solution, or even feasible. 


\subsection{THREE INTERSECTING FLOWS OF AIRCRAFT}

We have presented a strategy for the analysis of the airspace demand of a stable system of multiple intersecting flows. Our strategy involves decomposing the system into a network of fundamentally stable elements, the conflict zones. We have also extended the results of previous work in the field to derive an estimate of the airspace demand of a single conflict zone, in preparation for the analysis of multiple flow systems. As an intermediate step to a more general approach, we now apply the combined framework (decomposition strategy and conflict zone model) to consider the analysis of a system of three flows entering a control space.

For three flows there are three conflict zones, and their arrangement leads to the formation of a geometrical structure characterized by three circles, each centered at one of the vertices of a triangle. The stable control space must encompass the entire system. From Section 3 we note that the conflict zone radii are determined by the flow directions, which are given to the problem. The lateral displacements determine the position and size of the triangle, but we note that selections of $\mathbf{d} \in \mathbb{R}^{3}$ produce similar triangles, differing only in scale and position (see Figure 5). Therefore, the placement of the conflict zones is highly constrained, and the sizes are completely predetermined.

The algorithmic approach to the $n=3$ problem is as follows: First, we consider hypothetical displacements $d_{1}^{\prime}=0, d_{2}^{\prime}=0$, and find $d_{3}^{\prime}$ that forms the feasible configuration achieving a minimal control space. Second, we determine the parameters, $\left\{x_{\mathrm{c}}, y_{\mathrm{c}}, R\right\}$, of the smallest circle encompassing that system configuration, according to an origin resulting at the intersection of flows 1 and 2 . 
The coordinate pair $\left(x_{\mathrm{c}}, y_{\mathrm{c}}\right)$ corresponds to the center of the encompassing circle, while $R$ is its radius. Finally, we translate the coordinate system to the center of the circle and modify the original displacements to obtain $d_{1}, d_{2}$, and $d_{3}$ and complete the solution.

\subsection{DETERMINING A MINIMIZING FLOW CONFIGURATION}

The approach for three flows seems somewhat backward from the abstract description of our approach in Section 2.2. There we proceeded by a search algorithm, finding smaller and smaller circles to host a non-empty set of feasible flow configurations. Here instead we go directly, as if by magic, to the minimizing configuration, and then calculate the smallest circle which can support it. We will argue loosely the case for our solution to the minimizing configuration. First, it seems intuitive that the minimal arrangement would occur at the horizon of some conflict zone overlap, otherwise the protected zones could be brought uniformly closer together. Then, because all triangular arrangements are similar that are generated by a selection of displacements, we suggest that we select the displacement $d_{3}^{\prime}$ in such a way as to produce the smallest scale triangle that can host the conflict zones without overlap. This rather intuitive result, the most compact arrangement, can be shown to be the minimizing arrangement, so our procedures are valid.

In the following procedures we explain the mechanism by which we find the most compact arrangement. We begin by introducing some notation. We label the support direction angles $\theta_{1}, \theta_{2}, \theta_{3}$ in ascending order such that

$$
0 \leq \theta_{1}<\theta_{2}<\theta_{3}<2 \pi
$$

Note that we assume no parallel or anti-parallel angles. We let a set of angles $\left\{\phi_{i}\right\}$ correspond to the angles subtended by successive flows:

$$
\begin{aligned}
\phi_{1} & =\theta_{3}-\theta_{2} \\
\phi_{2} & =\theta_{1}+2 \pi-\theta_{3} \\
\phi_{3} & =\theta_{2}-\theta_{1} .
\end{aligned}
$$


Note that $\left\{\phi_{i}\right\}$ are defined circularly, as is apparent from the definition of $\phi_{2}$. That is, they are measured in an anti-clockwise manner, such that their sum is $2 \pi$, or a complete revolution. We denote $e_{1}, e_{2}, e_{3}$ the lengths of edges of the triangle formed from the intersections of the three flows (Figure 5). In addition, we denote $\left(x_{1}, y_{1}\right)$ and $r_{1}$ the center and radius, respectively, of the conflict zone surrounding the intersection between flows 2 and 3, and similarly use $\left(x_{2}, y_{2}\right)$ and $r_{2}$ to characterize the conflict zone of flows 3 and 1 , and $\left(x_{3}, y_{3}\right)$ and $r_{3}$ for the conflict zone of flows 1 and 2. Correspondingly, we denote positional vectors $\mathbf{x}_{i}=\left(x_{i}, y_{i}\right)^{T}$ for $i=1,2,3$.

From $\left\{\phi_{i}\right\}$ and (3.3), we determine the conflict zone radii by

$$
r_{i}=\frac{b D_{\mathrm{sep}}}{\sin \left(\phi_{i} / 2\right)}, \quad i=1,2,3
$$

From $\left\{\theta_{i}\right\}$ and $\left\{e_{i}\right\}$, we can derive the expressions for the intersections of the three flows:

$$
\begin{aligned}
& \mathbf{x}_{1}=\left(-e_{2} \sin \theta_{2}, e_{2} \cos \theta_{2}\right)^{T} \\
& \mathbf{x}_{2}=\left(-e_{1} \sin \theta_{1}, e_{1} \cos \theta_{1}\right)^{T} \\
& \mathbf{x}_{3}=(0,0)^{T} .
\end{aligned}
$$

Note that the third term is $(0,0)^{T}$, and this is because, according to the convention mentioned at the beginning of Section 4, the origin of the current coordinate system is located at the intersection of flows 1 and 2 .

As mentioned, the three segments, formed between pairs of flow intersections, constitute a triangle with conflict zones occurring at the vertices. We calculate the inner angles, $\left\{\alpha_{i}\right\}$, of the triangle that is formed. Figure 5 (left) and (right) show two disjoint possibilities for $\left\{\phi_{i}\right\}$ which lead to two separate cases for the solution of the inner angles. Figure 5 (left) shows the case where the orientations of all three flows can be seen to lie in a half-plane. The condition satisfied by this case is that one of the circularly defined $\phi_{i}>\pi$. The example in Figure 5 (left) shows $\phi_{2}$ as the culprit. Figure 5 (right) then, shows the other case, for which no half-plane contains all three flow directions. For clarification, the case in Figure 5 (left) is more likely than the other case because this case is more probable for $\theta_{1}, \theta_{2}, \theta_{3}$, a 

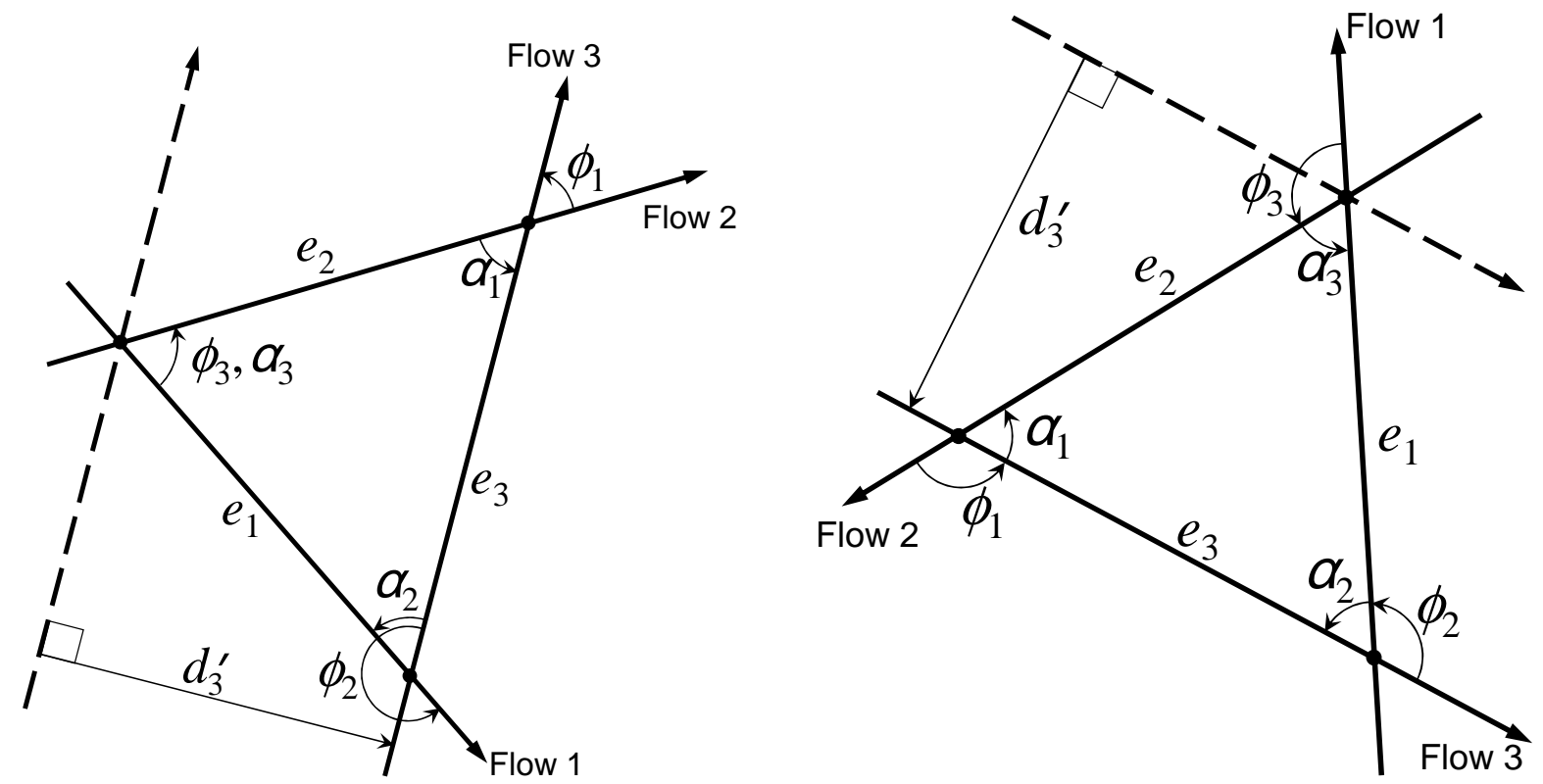

Figure 5: Two cases of a three-flow triangular flow configuration. (Left) Flow orientations are grouped into a half-plane. (Right) Flow orientations cannot be grouped into a half-plane.

sorted selection of three angles independently identically distributed, uniform over $[0,2 \pi)$. From inspection of Figure 5 (left) and (right) we find that $\left\{\alpha_{i}\right\}$ is given by

$$
\alpha_{i}= \begin{cases}\phi_{i}-\pi & \text { if } \phi_{i}>\pi \\ \pi-\phi_{i} & \text { if } \phi_{j}<\pi \text { for all } j \in\{1,2,3\} \\ \phi_{i} & \text { otherwise }\end{cases}
$$

where $i=1,2,3$.

In order to determine the most compact flow arrangement, we apply the Law of Sines. Using the result of (4.5) we can calculate the edge lengths $\left\{e_{i}\right\}$ of the triangle edges and complete the triangle parameterization. Note that, by Law of Sines, we can choose a number E such that

$$
E=\frac{e_{1}}{\sin \alpha_{1}}=\frac{e_{2}}{\sin \alpha_{2}}=\frac{e_{3}}{\sin \alpha_{3}} .
$$


The feasibility constraints for the triangle edge lengths $e_{1}, e_{2}, e_{3}$, to prevent conflict zone overlap, are as follows

$$
\begin{aligned}
& e_{1}=E \sin \alpha_{1} \geq r_{2}+r_{3} \\
& e_{2}=E \sin \alpha_{2} \geq r_{1}+r_{3} \\
& e_{3}=E \sin \alpha_{3} \geq r_{1}+r_{2}
\end{aligned}
$$

where $r_{i}$ is determined by (4.3) for $i=1,2,3$. Isolating $E$ on the left-hand side, we see that the smallest value which satisfies all three constraints simultaneously is given by

$$
E=\max \left\{\frac{r_{2}+r_{3}}{\sin \alpha_{1}}, \frac{r_{1}+r_{3}}{\sin \alpha_{2}}, \frac{r_{1}+r_{2}}{\sin \alpha_{3}}\right\}
$$

Based on (4.6) and (4.7), the triangle edge lengths $e_{1}, e_{2}, e_{3}$ under the most compact arrangement are determined by

$$
e_{i}=\max \left\{\frac{r_{2}+r_{3}}{\sin \alpha_{1}}, \frac{r_{1}+r_{3}}{\sin \alpha_{2}}, \frac{r_{1}+r_{2}}{\sin \alpha_{3}}\right\} \cdot \sin \alpha_{i}
$$

for $i=1,2,3$.

With all triangle parameters at our disposal, we now move to calculate the last relative displacement $d_{3}^{\prime}$. We will use this relative displacement to calculate the actual flow displacements according to the origin of the determined control space. Note that by our choice of $d_{1}^{\prime}$ and $d_{2}^{\prime}$ we have let the origin come to lie temporarily on the intersection between flows 1 and 2. We can see then, from either Figure 5 (left) or (right), that $d_{3}^{\prime}$ is given by

$$
d_{3}^{\prime}=e_{1} \sin \alpha_{2}=e_{2} \sin \alpha_{1}
$$

Once we find the circle parameters by means explored in the next subsection, we have the means to adjust the relative displacements found above. Specifically, for a circle center $\left(x_{\mathrm{c}}, y_{\mathrm{c}}\right)$ and the original line $\left\{\theta_{i}, d_{i}^{\prime}\right\}$, the adjusted, or actual, displacement $d_{i}$ is derived as follows. We apply (2.2) to a point on the flow line referenced from the new center, $\mathbf{x}_{\mathrm{c}}=$ $\left(x_{\mathrm{c}}, y_{\mathrm{c}}\right)^{T}$, of the control space (Figure 6): $d_{i}=\mathbf{u}_{i}^{T}\left(\mathbf{x}-\mathbf{x}_{\mathrm{c}}\right)$. Then we have $d_{i}=\mathbf{u}_{i}^{T} \mathbf{x}-\mathbf{u}_{i}^{T} \mathbf{x}_{\mathrm{c}}$, i.e.,

$$
d_{i}=d_{i}^{\prime}-\mathbf{u}_{i}^{T}\left[\begin{array}{l}
x_{\mathrm{c}} \\
y_{\mathrm{c}}
\end{array}\right] .
$$




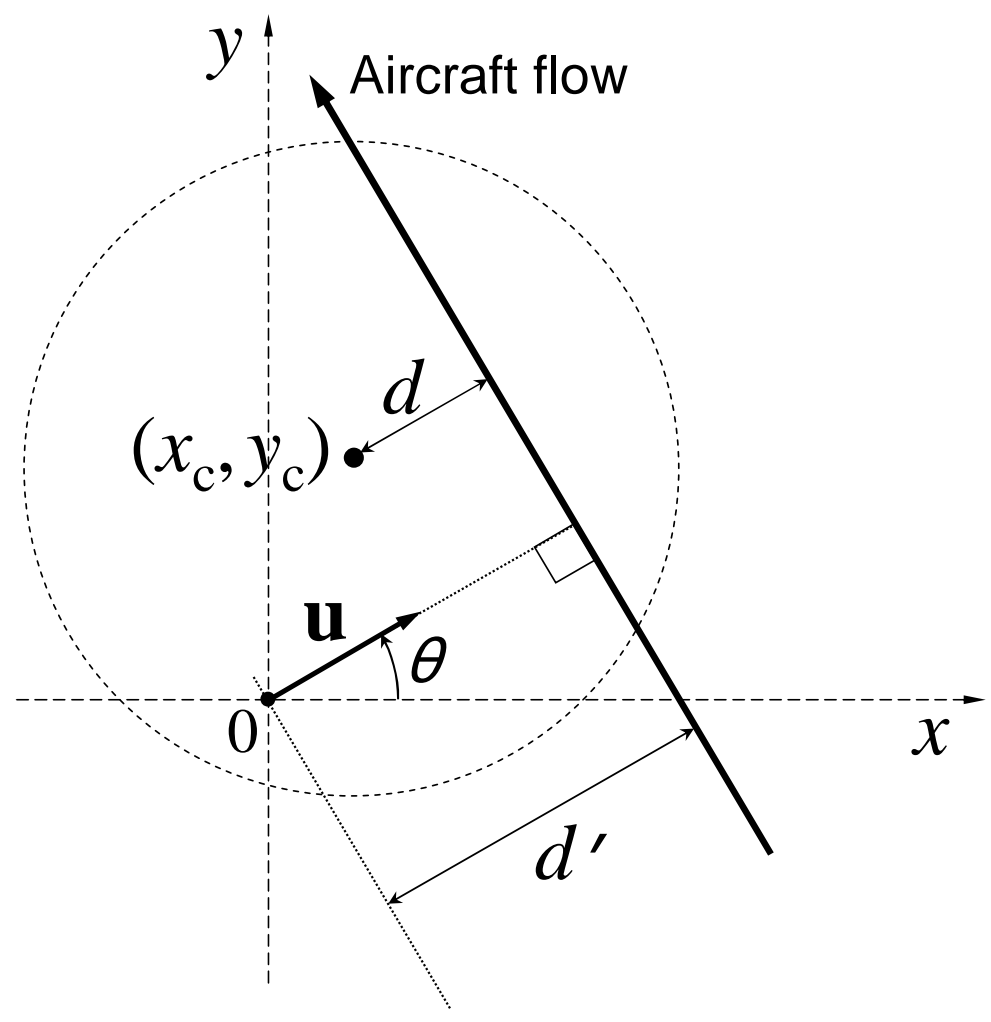

Figure 6: Determining re-parameterization of a flow given a new reference point. 


\subsection{ALIGNING A CIRCULAR REGION TO A MINIMAL CONFIGURATION}

We turn now to the problem remaining, of obtaining the minimal control space parameters, given a minimizing arrangement. For the three-flow problem, we will provide a general solution for the smallest circle encompassing three arbitrary circles (conflict zones), so that we may apply it to the minimizing arrangement found in Section 4.1 above. We begin by presenting a proposition regarding the smallest encompassing circle. The proof of this proposition is given in the appendix.

Proposition 1. The circle of least area encompassing three arbitrary circles must satisfy one of the following two conditions: (Condition 1) That circle corresponds to the minimal circle encompassing a selection of two out of the three circles - the selection producing the largest minimal circle-and the third circle remains inside. (Condition 2) The minimal encompassing circle is tangent to all three inner circles, and therefore corresponds to the smaller of at least one, and at most two, solutions to the Apollonius' Circle Problem [21] satisfying outer tangency with all three circles.

In the following we investigate the two conditions of Proposition 1, respectively.

\subsubsection{Minimal Configuration Touches Two Interior Circles}

Consider that the minimal circle encompassing a selection of two out of the three circles may suffice to encompass all three. Because such a circle is never larger than one which encompasses all three circles, we test this case first. By Lemma 2 (see the appendix) and Condition 1 of Proposition 1, we can arrive at an expression for the radius of the candidate circle:

$$
R=\frac{1}{2} \max \left\{e_{1}+r_{2}+r_{3}, e_{2}+r_{1}+r_{3}, e_{3}+r_{1}+r_{2}\right\}
$$

Certainly this circle (see Figure 7) contains the two circles to whom it is tangent on the outer edge, but we must show whether this circle contains the third circle, which it does not necessarily touch. 
Note that it is impossible for any but the largest circle drawn tangent to the outer edges of two out of the three circles to contain all three. This is the reason we choose the largest distance in (4.11).

We can test whether the third circle is contained through the following method. Without loss of generality, let us assume that it is $e_{3}$ which connects the circles whose outer edges are farthest apart [maximizes (4.11)]. That is, we assume that we chose

$$
R=\frac{e_{3}+r_{1}+r_{2}}{2}
$$

Analysis of Figure 7 allows us to solve the distance from the center of the encompassing circle to the outer edge of the uncertain inner circle:

$$
R_{\mathrm{d}}=r_{3}+l_{3}
$$

where $R_{\mathrm{d}}$ is the demand of the third circle and $l_{3}$ is the distance from the center of the encompassing circle to the center of the third inner circle:

$$
l_{3}=\sqrt{\left(R-r_{1}\right)^{2}+e_{2}^{2}-2 e_{2}\left(R-r_{1}\right) \cos \alpha_{1}} .
$$

From this the test is quite simple: If $R \geq R_{\mathrm{d}}$ then the circle is included (has slack).

If the candidate circle does encompass the third circle, then we must locate the control space center at a position $\mathbf{x}_{\mathbf{c}}$ according to the temporary coordinate system. If we assume, as we have done above, that circle $1,\left\{\mathbf{x}_{1}, r_{1}\right\}$, and circle $2,\left\{\mathbf{x}_{2}, r_{2}\right\}$-connected by an edge of length $e_{3}$ - are the circles tangent to the minimal encompassing circle, then we can derive the control-space center as follows:

$$
\mathbf{x}_{\mathrm{c}}=\frac{\left[e_{3}-\left(r_{2}-r_{1}\right)\right] \mathbf{x}_{1}+\left[e_{3}+\left(r_{2}-r_{1}\right)\right] \mathbf{x}_{2}}{2 e_{3}}
$$

where $\mathbf{x}_{i}$ can be determined by (4.4) and (4.8). Note that if another edge is chosen to maximize (4.11), then the appropriate juxtaposition in the terms of (4.12) and (4.13) must be made. 


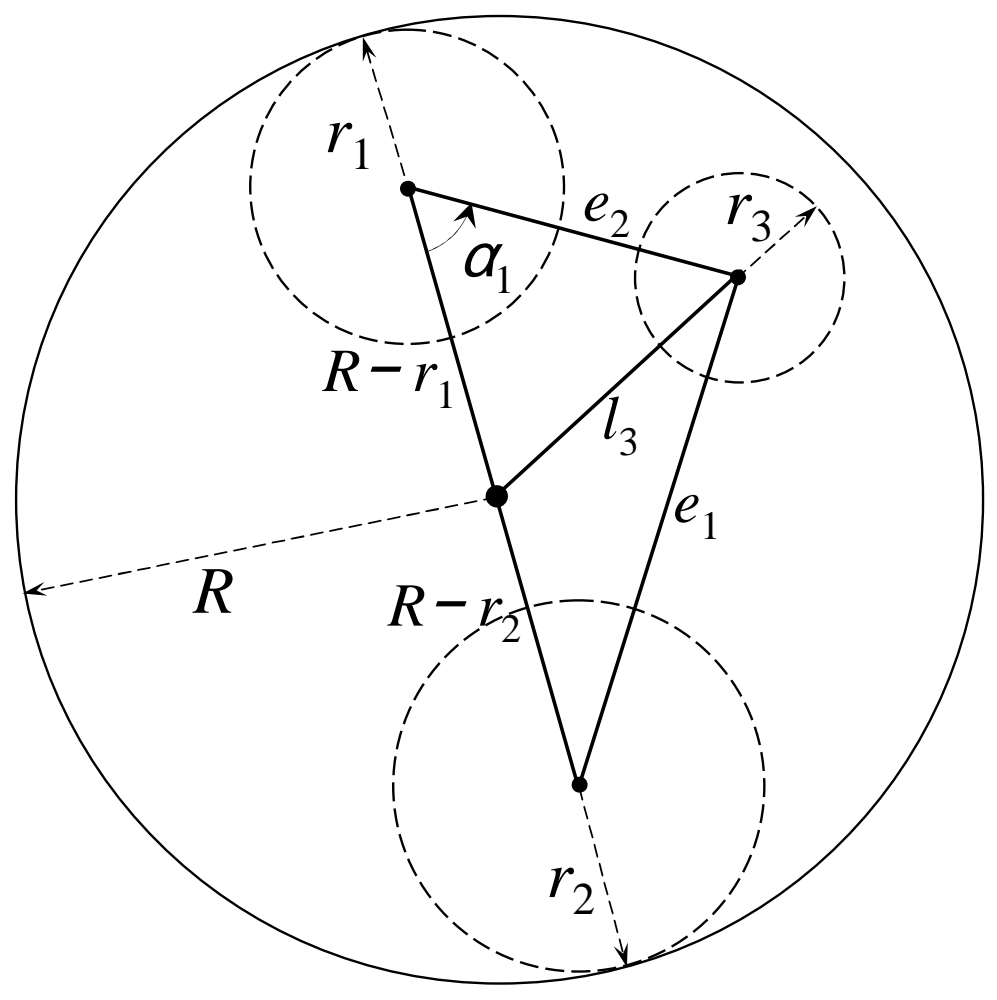

Figure 7: Determining the slack allowed for a third circle given tangency to two of an encompassing circle of arbitrary radius. 


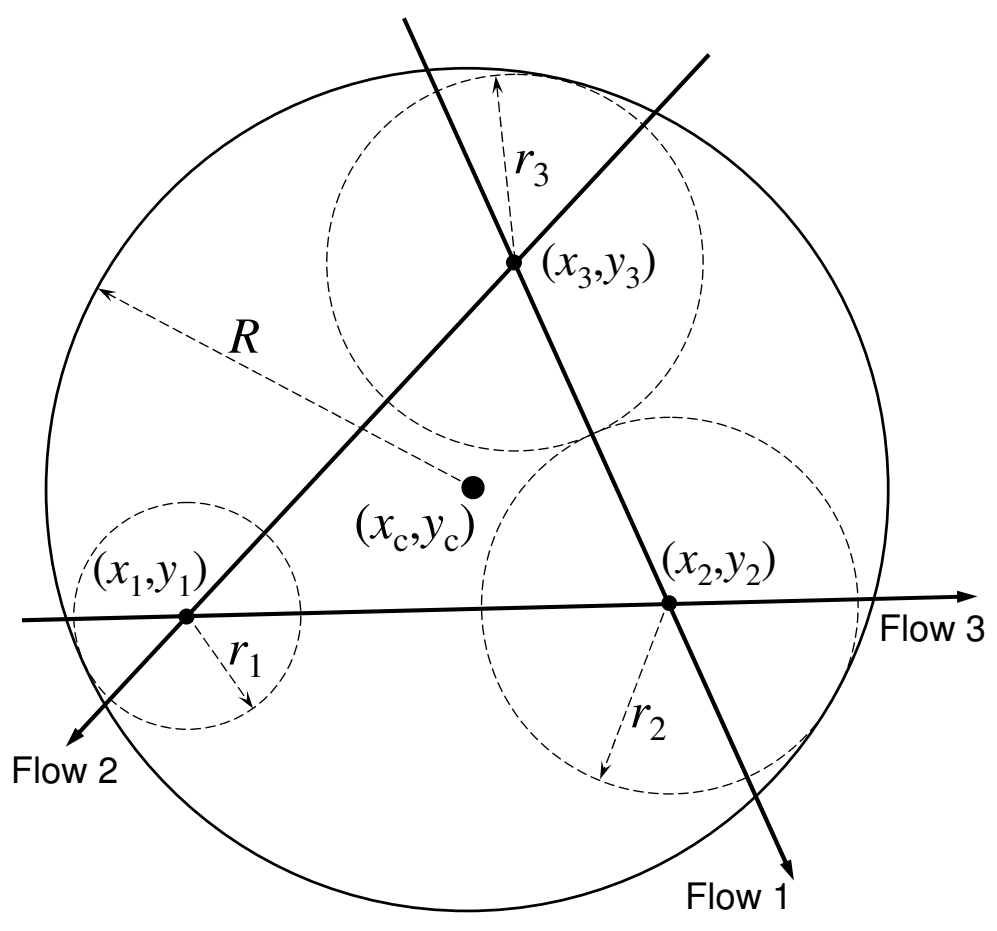

Figure 8: Apollonius' circle, the encompassing circle tangent to all three inner circles. 


\subsubsection{Minimal Configuration Touches Three Interior Circles}

If the third circle is over demanding, then the minimal feasible circle touches all three inner circles, as dictated by Proposition 1. The minimal arrangement and control space therefore satisfy the Apollonius' circle solution with outer tangency [21]. This outer tangency is described by a system of equations, which we derive from inspection of Figure 8:

$$
\left(x_{i}-x_{\mathrm{c}}\right)^{2}+\left(y_{i}-y_{\mathrm{c}}\right)^{2}-\left(R-r_{i}\right)^{2}=0, \quad i=1,2,3
$$

where $x_{i}$ and $y_{i}$ are determined by (4.4) and (4.8) and $r_{i}$ is determined by (4.3). As long as the circle centers are not collinear, this system can produce $x_{\mathrm{c}}$ and $y_{\mathrm{c}}$ in terms of $R$. Luckily, by our previous assumptions of non-parallel angles we have ruled out this exceptional possibility. Therefore we can find the circle center $\left(x_{\mathrm{c}}, y_{\mathrm{c}}\right)$ in terms of $R$ :

$$
\begin{aligned}
& x_{\mathrm{c}}=\gamma_{x} R+\beta_{x} \\
& y_{\mathrm{c}}=\gamma_{y} R+\beta_{y}
\end{aligned}
$$

where

$$
\begin{aligned}
\gamma_{x} & =\left[\left(y_{2}-y_{1}\right)\left(r_{3}-r_{1}\right)-\left(y_{3}-y_{1}\right)\left(r_{2}-r_{1}\right)\right] /\left[\left(x_{2}-x_{1}\right)\left(y_{3}-y_{1}\right)-\left(x_{3}-x_{1}\right)\left(y_{2}-y_{1}\right)\right] \\
\beta_{x} & =\frac{\left(x_{1}^{2}+y_{1}^{2}-r_{1}^{2}\right)\left(y_{2}-y_{3}\right)+\left(x_{2}^{2}+y_{2}^{2}-r_{2}^{2}\right)\left(y_{3}-y_{1}\right)+\left(x_{3}^{2}+y_{3}^{2}-r_{3}^{2}\right)\left(y_{1}-y_{2}\right)}{2\left(x_{2}-x_{1}\right)\left(y_{3}-y_{1}\right)-2\left(x_{3}-x_{1}\right)\left(y_{2}-y_{1}\right)} \\
\gamma_{y} & =\left[\left(x_{3}-x_{1}\right)\left(r_{2}-r_{1}\right)-\left(x_{2}-x_{1}\right)\left(r_{3}-r_{1}\right)\right] /\left[\left(x_{2}-x_{1}\right)\left(y_{3}-y_{1}\right)-\left(x_{3}-x_{1}\right)\left(y_{2}-y_{1}\right)\right] \\
\beta_{y} & =\frac{\left(x_{1}^{2}+y_{1}^{2}-r_{1}^{2}\right)\left(x_{3}-x_{2}\right)+\left(x_{2}^{2}+y_{2}^{2}-r_{2}^{2}\right)\left(x_{1}-x_{3}\right)+\left(x_{3}^{2}+y_{3}^{2}-r_{3}^{2}\right)\left(x_{2}-x_{1}\right)}{2\left(x_{2}-x_{1}\right)\left(y_{3}-y_{1}\right)-2\left(x_{3}-x_{1}\right)\left(y_{2}-y_{1}\right)} .
\end{aligned}
$$

Then we can substitute these expressions into one of the equations of (4.14) and achieve a quadratic equation solvable in $R$. Finally we obtain

$$
R=\frac{-b_{R} \pm \sqrt{b_{R}^{2}-4 a_{R} c_{R}}}{2 a_{R}}
$$

where

$$
\begin{aligned}
a_{R} & =\gamma_{x}^{2}+\gamma_{y}^{2}-1 \\
b_{R} & =2\left[\gamma_{x}\left(\beta_{x}-x_{i}\right)+\gamma_{y}\left(\beta_{y}-y_{i}\right)+r_{i}\right] \\
c_{R} & =\left(\beta_{x}-x_{i}\right)^{2}+\left(\beta_{y}-y_{i}\right)^{2}-r_{i}^{2} .
\end{aligned}
$$

Naturally, we choose the smallest positive $R$ as the radius of the minimal solution. 
Negative solutions correspond to Apollonius' circles satisfying inner tangency, and we do not consider the possibility of complex roots because they can only occur when the Apollonius' circle is not the minimal circle.

With this analysis and final result, we have completed our closed-form solution, both for the minimal control space and the minimizing configuration for three intersecting flows. 


\subsection{CONSIDERATIONS FOR MULTIPLE FLOWS OF AIRCRAFT}

We have explored procedures for determining the complexity of systems of two and three flows. In this section we will consider the increasing complexity of the multiple flow problem with numbers of air traffic flows larger than $n=3$. We will begin by formulating an optimization problem whose solution, if we can find it, determines the smallest control space (with radius $R_{\text {min }}$ ) for which we can host $n$ flows at arbitrary (non-parallel) angles with guaranteed stability. Unfortunately, the problem contains non-convex constraints and is difficult to solve in general. We will continue by examining the growing complexity associated with an $n$-flow system possessed of some symmetrical structure. We will seek some trend in the growth of $R_{\min }$.

\subsection{GENERAL OPTIMIZATION PROBLEM}

We wish to find the smallest circle that can host all conflict zones without overlap. We can formulate this problem as an optimization problem, and at first glance the objective function to minimize seems simple enough: It is $R$, the radius of the ultimate control space. The constraints however can be numerous. The following analysis is illustrated by the situation depicted in Figure 2.

As is the nature of the problem, the constraints largely consider the location and size of conflict zones. For $n$ flows, there are $X=n(n-1) / 2$ intersections or conflict zones. Therefore, there are $S=X(X-1) / 2$ constraints from the pairwise combination of conflict zones (any pair of conflict zones should not overlap). These $S$ constraints will be referred to as separations or internal constraints. As introduced previously, we use the multi-dimensional 
vector $\mathbf{d}=\left(d_{1}, \ldots, d_{n}\right)^{T}$ to represent the lateral flow displacements, which directly affect the locations of intersections. It may be useful to consider that, as $n$ grows larger, the activity of these internal constraints becomes sparse for a feasible solution. That is, the ratio of active separations (satisfied with equality) to total separations decreases with increasing number of flows. Denote $\mathbf{x}_{j k}=\left(x_{j k}, y_{j k}\right)^{T}$ the point of intersection occurring between flows $j$ and $k$, and denote $r_{j k}$ the radius of the conflict zone centered at $\mathbf{x}_{j k}$. Then any pair of conflict zones can be characterized by $\left\{\mathbf{x}_{j k}, r_{j k}\right\}$ and $\left\{\mathbf{x}_{j^{\prime} k^{\prime}}, r_{j^{\prime} k^{\prime}}\right\}$ with (i) $1 \leq j<k \leq n$, (ii) $1 \leq j^{\prime}<k^{\prime} \leq n$, and (iii) $j \neq j^{\prime}$ or $k \neq k^{\prime}$. Such a pair of conflict zones yields an internal constraint of the following form:

$$
\left(x_{j k}-x_{j^{\prime} k^{\prime}}\right)^{2}+\left(y_{j k}-y_{j^{\prime} k^{\prime}}\right)^{2} \geq\left(r_{j k}+r_{j^{\prime} k^{\prime}}\right)^{2} .
$$

The above internal constraints can be expressed in terms of the decision variables $d_{1}, \ldots, d_{n}$ (or decision-variable vector $\mathbf{d}$ ) and the given flow orientations $\theta_{1}, \ldots, \theta_{n}$. Recall that the position $\left(x_{j k}, y_{j k}\right)$ is a function of $d_{j}$ and $d_{k}$. Specifically, $\left(x_{j k}, y_{j k}\right)$ satisfies the line equations for flows $j$ and $k$ [see (2.2) and (2.1)] simultaneously:

$$
\left[\begin{array}{l}
d_{j} \\
d_{k}
\end{array}\right]=\left[\begin{array}{c}
\mathbf{u}_{j}^{T} \\
\mathbf{u}_{k}^{T}
\end{array}\right]\left[\begin{array}{l}
x_{j k} \\
y_{j k}
\end{array}\right]=\left[\begin{array}{cc}
\cos \theta_{j} & \sin \theta_{j} \\
\cos \theta_{k} & \sin \theta_{k}
\end{array}\right]\left[\begin{array}{l}
x_{j k} \\
y_{j k}
\end{array}\right] .
$$

Therefore, we can derive

$$
\begin{aligned}
x_{j k} & =\frac{\sin \theta_{k}}{\sin \left(\theta_{k}-\theta_{j}\right)} d_{j}-\frac{\sin \theta_{j}}{\sin \left(\theta_{k}-\theta_{j}\right)} d_{k} \\
y_{j k} & =-\frac{\cos \theta_{k}}{\sin \left(\theta_{k}-\theta_{j}\right)} d_{j}+\frac{\cos \theta_{j}}{\sin \left(\theta_{k}-\theta_{j}\right)} d_{k} .
\end{aligned}
$$

Furthermore, according to $(3.3), r_{j k}$ is determined by

$$
r_{j k}=\frac{b D_{\mathrm{sep}}}{\sin \frac{\theta_{k}-\theta_{j}}{2}}
$$

Recall that the flows are indexed in ascending order of the angle $\theta_{i} \in[0,2 \pi)$ for $i=1, \ldots, n$, so $\left(\theta_{k}-\theta_{j}\right) / 2$ remains within $(0, \pi)$ for $j<k$ - this guarantees that the right-hand side of (5.3) is strictly greater than 0 . 
The internal constraints guarantee separation of a flow configuration parameterized by d. However, a second class of constraints is required, which forms a set of lower bounds on the area of the control space. This smaller set of constraints, the containments or external constraints, has elements in the following form:

$$
x_{j k}^{2}+y_{j k}^{2} \leq\left(R-r_{j k}\right)^{2}, \quad R \geq r_{j k}
$$

where $x_{j k}, y_{j k}$, and $r_{j k}$ can be expressed in terms of $d_{j}, d_{k}, \theta_{j}$, and $\theta_{k}$, using (5.2) and (5.3). These external constraints guarantee that all conflict zones are within the control space, and form a constraint mainly of $R$.

With these two classes of constraints we can present the optimization problem for finding the smallest circle that hosts all conflict zones without overlap:

$$
\begin{aligned}
\text { minimize } & R \\
\text { subject to } & (5.1) \text { and }(5.4) \text { for } \\
& 1 \leq j<k \leq n, 1 \leq j^{\prime}<k^{\prime} \leq n \text {, and }\left(j \neq j^{\prime} \text { or } k \neq k^{\prime}\right) .
\end{aligned}
$$

Note the motivation behind this formulation from the standpoint of the abstract language developed in Section 2.2. The two types of constraints, (5.1) and (5.4), can be considered the conditions of feasibility for $\mathbf{d}$. That is, we can now provide a complete description of the set $\mathcal{D}_{\text {feas }}$ :

$$
\mathcal{D}_{\text {feas }}(\boldsymbol{\theta}, R)=\{\mathbf{d} \mid(5.1) \text { and }(5.4)\}
$$

Then the same optimization problem can also be formulated as follows: minimize $R$ over $\mathbf{d}$ and $R$ subject to $\mathbf{d} \in \mathcal{D}_{\text {feas }}(\boldsymbol{\theta}, R)$. We see that this expression is intuitively equivalent to the approach we have already described.

Of course, this optimization problem is difficult to solve, because it involves non-convex constraints. Note, however, that the $n=3$ solution analyzed previously is a special case of this very optimization formulation: The property of the $n=3$ problem which allows us to solve the optimization problem in a closed-form manner is that we can find a set of displacements $\mathbf{d}$ (most compact) to achieve the minimizing feasible arrangement of three conflict zones. 

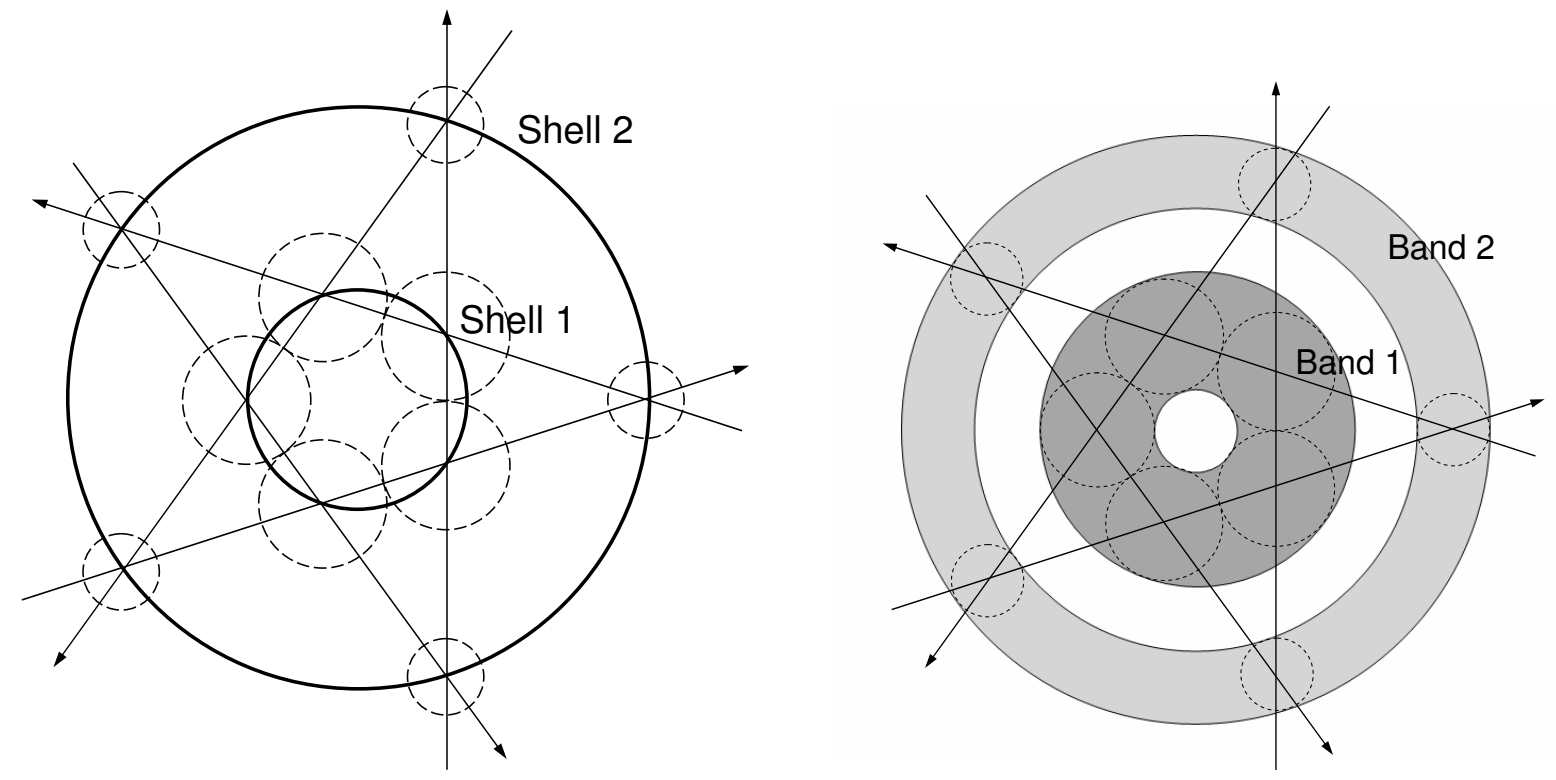

Figure 9: (Left) The symmetric $n$-flow shell definition. (Right) The symmetric $n$-flow band definition.

\subsection{HOW TRAFFIC COMPLEXITY IS AFFECTED BY NUMBER OF FLOWS}

In previous sections we have developed a formal approach to a measure of complexity, or a condition of stability, for a system of air traffic flows. In this section we want to examine the growth in demand of area for stability with increasing numbers of flows. This examination should provide insight into the usefulness of the current model, and where it might be improved. We consider a control space with $n$ symmetric flows, i.e., $n$ flow orientations evenly spaced over the circumference of the control space:

$$
\theta_{i}=\frac{2 \pi}{n}(i-1), \quad i=1, \ldots, n
$$

Even for a symmetric arrangement, the optimization formulation for the problem is difficult to solve. However, in establishing an upper bound on the demand for stability we consider a symmetric configuration of flows, which is achieved by setting $d_{i}=d$ for any $i$. 
As a result of the symmetric configuration, we find that the points of intersection of flows, if treated as vertices, form a sequence of regular $n$-gons in shells about the origin [see Figure 9 (left)]. In general, the $n$-gon shells can be indexed by $k$, where the $k$-th shell is formed by the $n$ intersections between flows $i=1, \ldots, n$ and $j=((i+k))_{n}$. Here the operator $((\cdot))_{m}$ implies modulo-m wrapping over the index range. The shell index $k$ itself is found in the range $k=1, \ldots, K$, where

$$
K=\left\lfloor\frac{n-1}{2}\right\rfloor
$$

i.e., the largest integer that is no greater than $(n-1) / 2$. The result expressed for $K$ accounts for the number of flows contained within the half circle $\theta_{i} \in(0, \pi)$, or all flows for which intersections with Flow $1\left(\theta_{1}=0\right)$, lie in distinct shells. Flows beyond $\theta_{i}=\pi$ intersect with Flow 1 on shells already formed with previous flows. Note that for even numbers of flows a $\theta_{i}=\pi$ is produced, which is not counted in $K$. This final would-be stage does not provide a shell in the finite plane because the flows are anti-parallel, and so do not intersect. As a result, there is no conflict, so the shell is simply ignored. Because of symmetry, we can express the angle between two flows intersecting in the $k$-th shell by $\phi^{(k)}$, and we can derive

$$
\phi^{(k)}=\frac{2 \pi k}{n} .
$$

For the symmetric system, all intersections within a shell are equidistant from the center of the space, and all conflict zones within a shell have the same radius. The dimensions of the frame of the system - that is, the network of flow segments formed between intersections - are proportional to the single displacement variable $d$. The problem to be solved is to minimize $d$ while ensuring that the frame supports all conflict zones without overlap. We denote $d_{\min }$ the solution to this problem.

Now if we consider, instead of shells, that the conflict zones are arranged in bands [Figure 9 (right)], then we can tighten the constraints slightly and still obtain a relatively tight upper bound on the demand for a symmetric flow configuration. We can easily find a $d$ that will prevent any bands from overlapping, instead of monitoring the inter-laying of conflict zones between shells. We use $d_{\text {upper }}$ to represent such a value of $d$, which is an upper bound of $d_{\min }$. 
Recall that the frame dimensions are proportional to $d$. We let the distance from the origin to a $k$-th shell intersection be expressed as $l^{(\mathrm{k})}=\lambda^{(\mathrm{k})} d$. Also, we let the radius of any circular conflict zone in shell $k$ be given by $r^{(k)}$, which we can calculate from $\phi^{(k)}$ and (3.3):

$$
r^{(\mathrm{k})}=\frac{b D_{\mathrm{sep}}}{\sin (\pi k / n)}
$$

Then for $K$ shells ( $K \geq 2$ and $n \geq 5$ ), a set of $K-1$ constraints exist such that a set of bands containing the shells entirely do not result in overlap: $l^{(\mathrm{k}+1)}-l^{(\mathrm{k})} \geq r^{(\mathrm{k}+1)}+r^{(\mathrm{k})}$, i.e.,

$$
d \geq \frac{r^{(\mathrm{k}+1)}+r^{(\mathrm{k})}}{\lambda^{(\mathrm{k}+1)}-\lambda^{(\mathrm{k})}}, \quad k=1, \ldots, K-1
$$

where $\lambda^{(\mathrm{k})}$ is calculated as follows. We simply evaluate the Cartesian distance established by the coordinates given in (5.2). Let $\theta_{j}=0$ and $\theta_{k}=\phi^{(k)}$, and finally $d_{j}=d_{k}=1$ (normalizing to $d$ ). Then we have

$$
\lambda^{(k)}=\left[\cos \left(\frac{\pi k}{n}\right)\right]^{-1} .
$$

By substituting (5.6) and (5.8) into (5.7), we obtain

$$
\begin{aligned}
& d \geq \frac{\frac{b D_{\mathrm{sep}}}{\sin [\pi(k+1) / n]}+\frac{b D_{\mathrm{sep}}}{\sin (\pi k / n)}}{\frac{1}{\cos [\pi(k+1) / n]}-\frac{1}{\cos (\pi k / n)}} \\
& =b D_{\text {sep }}\left\{\frac{\cos [\pi(k+1) / n] \cos (\pi k / n)}{\sin [\pi(k+1) / n] \sin (\pi k / n)}\right\}\left\{\frac{\sin [\pi(k+1) / n]+\sin (\pi k / n)}{\cos (\pi k / n)-\cos [\pi(k+1) / n]}\right\} \\
& =b D_{\operatorname{sep}}\left\{\frac{1}{\tan [\pi(k+1) / n] \tan (\pi k / n)}\right\}\left\{\frac{2 \sin [\pi(2 k+1) /(2 n)] \cos [\pi /(2 n)]}{2 \sin [\pi(2 k+1) /(2 n)] \sin [\pi /(2 n)]}\right\} \\
& =\frac{b D_{\mathrm{sep}}}{\tan [\pi(k+1) / n] \tan (\pi k / n) \tan [\pi /(2 n)]}, \quad k=1, \ldots, K-1 .
\end{aligned}
$$

Note that in the above expression the right-hand side achieves its maximum when $k=1$, so we can replace the above $K-1$ constraints with a single constraint, the constraint for $k=1$ :

$$
d \geq \frac{b D_{\text {sep }}}{\tan (2 \pi / n) \tan (\pi / n) \tan [\pi /(2 n)]} .
$$

One additional constraint also exists, a "string of pearls" constraint, which requires the inner-most shell to accommodate all $n$ flows without overlap of conflict zones within the band (Figure 10). This constraint is sufficient for overlap within all shells because the shells 


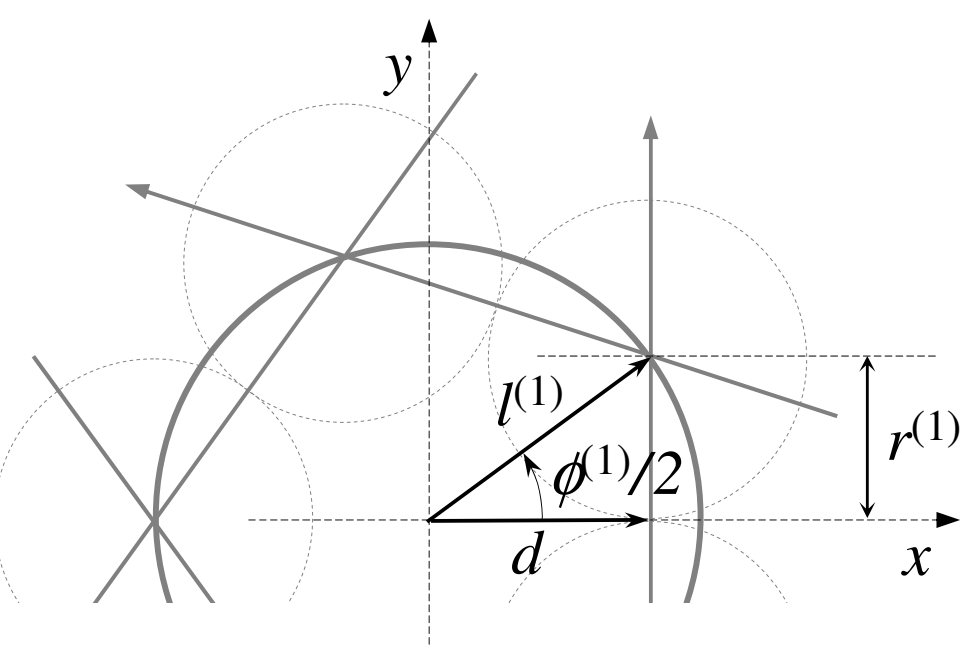

Figure 10: The string of pearls constraint.

further out have larger circumference and smaller conflict zone radius: $l^{(1)} \sin (\pi / n) \geq r^{(1)}$, i.e.,

$$
d \geq \frac{r^{(1)}}{\lambda^{(1)} \sin (\pi / n)}=\frac{b D_{\mathrm{sep}} \cos (\pi / n)}{[\sin (\pi / n)]^{2}} .
$$

Therefore, $d_{\text {upper }}$ is the solution to the problem of minimizing $d$ subject to the constraints of (5.9) and (5.10). In other words,

$$
d_{\text {upper }}=\max \left\{\frac{b D_{\text {sep }}}{\tan (2 \pi / n) \tan (\pi / n) \tan [\pi /(2 n)]}, \frac{b D_{\text {sep }} \cos (\pi / n)}{[\sin (\pi / n)]^{2}}\right\} .
$$

It can be verified that the first term of the right-hand side dominates for $n \geq 6$, and as $n$ goes to $\infty$ we have the following result (by using the fact $\lim _{x \rightarrow 0}(\tan x) / x=1$ )

$$
\lim _{n \rightarrow \infty} \frac{d_{\text {upper }}}{n^{3}}=\frac{b D_{\text {sep }}}{\pi^{3}}
$$

which implies $d_{\text {upper }} \in \Theta\left(n^{3}\right)$ (meaning that $d_{\text {upper }}$ is within a constant multiple of $n^{3}$ ).

In order to show that the band-constrained upper bound is relatively tight, we will find a lower bound of $d_{\min }$ that is of the same order. We can achieve such a lower bound, denoted $d_{\text {lower }}$, by noting that odd sequenced shells $(1,3,5, \ldots$, or $2,4,6, \ldots)$ are oriented in the same way. That is, the vertices in the regular $n$-gons of the odd sequence occur along the same 
radial lines (here $n$ should be no less than 7 so as to ensure $K \geq 3$ ). As a result, if bands 1 and 3 are brought together, it implies tangency of the conflict zones between the bands. Minimally satisfying this constraint alone provides a lower bound on $d_{\min }$ for a symmetric flow configuration:

$$
d_{\text {lower }}=\frac{r^{(3)}+r^{(1)}}{\lambda^{(3)}-\lambda^{(1)}}=\frac{b D_{\text {sep }}}{\tan (3 \pi / n)[\tan (\pi / n)]^{2}} .
$$

It can be verified that

$$
\lim _{n \rightarrow \infty} \frac{d_{\text {lower }}}{n^{3}}=\frac{b D_{\text {sep }}}{3 \pi^{3}}
$$

which implies $d_{\text {lower }} \in \Theta\left(n^{3}\right)$. Since both $d_{\text {upper }}$ and $d_{\text {lower }}$ belong to $\Theta\left(n^{3}\right)$, it has to be true that $d_{\text {min }}$ belongs to $\Theta\left(n^{3}\right)$ as well.

In our simulations (not shown here), an interesting finding is that for $n \geq 16$ flows, the tangency of bands 1 and 3 becomes sufficient to prevent conflict zone overlap. As a result, the actual demand in lateral displacement of flows for a symmetric configuration corresponds to (is not simply bounded by) this constructed lower bound $d_{\text {lower }}$ for $n$ sufficiently large.

In the above we have determined the growth in demand of the flow-configuration parameter $d$ with increasing numbers of flows. Finally, we study the growth of the airspace demand for a symmetric configuration of $n$ flows as $n$ increases. The control space radius shares the outer edge of the $K$-th band: $R=l^{(K)}+r^{(K)}=\lambda^{(K)} d+r^{(K)}$. Therefore,

$$
R_{\min }=\lambda^{(K)} d_{\min }+r^{(K)}
$$

which is bounded from above by $\lambda^{(K)} d_{\text {upper }}+r^{(K)}$ and bounded from below by $\lambda^{(K)} d_{\text {lower }}+r^{(K)}$. Note that

$$
\lim _{n \rightarrow \infty} \frac{\lambda^{(K)}}{n}=\lim _{n \rightarrow \infty} \frac{1}{n \cos (\pi K / n)}=\lim _{n \rightarrow \infty} \frac{1}{n(\pi / 2-\pi K / n)}= \begin{cases}2 / \pi & \text { if } n \text { is odd } \\ 1 / \pi & \text { if } n \text { is even }\end{cases}
$$

Furthermore, as $n$ goes to $\infty$, the $K$-th shell radius $r^{(K)}$ approaches $b D_{\text {sep }}$, and therefore $\lim _{n \rightarrow \infty} r^{(K)} / n=0$. So we have

$$
\lim _{n \rightarrow \infty} \frac{R_{\min }}{n^{4}} \leq \lim _{n \rightarrow \infty} \frac{d_{\text {upper }}}{n^{3}} \frac{\lambda^{(K)}}{n} \leq \frac{2 b D_{\text {sep }}}{\pi^{4}}
$$


and

$$
\lim _{n \rightarrow \infty} \frac{R_{\min }}{n^{4}} \geq \lim _{n \rightarrow \infty} \frac{d_{\text {lower }}}{n^{3}} \frac{\lambda^{(K)}}{n} \geq \frac{b D_{\text {sep }}}{3 \pi^{4}}
$$

implying that $R_{\text {min }}$ belongs to $\Theta\left(n^{4}\right)$. The order of growth of the demand for control space area then, for symmetric configurations, is $\Theta\left(n^{8}\right)$.

Note that the order of growth of the demand for airspace for stability (a measure of the air traffic complexity) is too high to be practical. It is encouraging that the demand exhibits polynomial growth, but the rate is too large. In additional to the large rate of growth, the actual demand is quite difficult to judge for any but the simplest of flow approaches. One solution, which is left for future work, may be to combine sets of flows in proximity to each other, into "thick" flows, and then re-evaluate the airspace demand. 


\subsection{CONCLUSIONS}

In this paper we have developed a general framework to study the conflict resolution for multiple intersecting flows of aircraft based on decomposition of the original problem into a sequence of two-flow problems. In the vicinity of the intersection between a pair of aircraft flows, we define the conflict zone as a circular area centered at the intersection such that aircraft approaching the intersection can resolve conflict completely within the conflict zone, without straying outside the zone. We consider the conflict zone as the fundamental stable unit of airspace for conflict resolution. Building upon the analytical results for two intersecting flows within a conflict zone, we estimate the performance bounds as well as safe space capacity for three and more intersecting flows. Our strategy is to displace the aircraft flows so that they intersect in pairs, instead of all at once, and so that the resulting conflict zones have no overlap. We formulate this problem into an optimization problem, which aims to keeps airspace demand (measured by the radius of a circular region encompassing all the conflict zones) as low as possible. The most compact airspace demand, or the radius of the smallest circle encompassing all conflict zones, provides an indication of the traffic complexity for multiple intersecting flows of aircraft. Such as indication is a function of the number and orientation of the aircraft flows entering the control space. Although the optimization problem to find the smallest control-space radius is difficult to solve in general, we have developed an algorithmic procedure to find a closed-form solution for the three-flow problem. For the numbers of flows greater than three, we have studied the symmetric configuration and shown that the order of growth of the demand for control-space radius is $\Theta\left(n^{4}\right)$ for $n$ flows of aircraft. 
In terms of the relation of this work to Histon's structural abstractions, we find that the adoption of multiple randomly intersecting flows of aircraft - at least to the extent that they are analyzed here - does not pose a reasonable compromise between computational complexity and organizational capacity. The rate of growth of airspace demand with increasing numbers of flows is prohibitively large in a system where airspace is the most coveted resource available. The contribution of this work is a comprehensive analysis of this type of structural abstraction - the arbitrary-density aircraft flow. We have also suggested that flow numbers (and therefore complexity) might be mitigated by recombining several near-parallel flows into "thick" flows, though we have left for future work the analysis of the degree to which complexity can be reduced in this way. The idea of structural abstraction remains a very relevant and important concern in proposed reform to the ATM system. Identification of structures that do provide a reasonable compromise between complexity and capacity will be crucial for the design of systems, both to foster important movements like Free Flight and to enable the progress of automation for mobile robotic vehicles as their utilization becomes more prevalent. Future work in this area should address the challenges of identifying such structures, to include 1) a systematic approach to identification, and 2) new analytical and computational methods for validating structural models. 


\section{APPENDIX}

\section{PROOF OF PROPOSITION}

We begin by presenting two lemmas.

Lemma 1. Consider three circles of arbitrary size and placement and the fourth circle drawn to contain all three inner circles. The circle of least area encompassing these three circles must be tangent from the inside to the surfaces of either two or three of the circles encompassed.

Proof. The number of circles tangent to the inner boundary of the encompassing circle is limited to the range $\{0,1,2,3\}$, since there are at most three circles. We seek to eliminate the possibilities apart from two or three tangent circles. We prove by contradiction. First, assume that the minimal encompassing circle touches no inner circles. Then by the argument of continuity we can always shrink the circle radius by some non-zero value while keeping all circles contained inside. This contradicts the assumption that the circle is minimal. Second, assume that the minimal encompassing circle touches one inner circle. Again, by the argument of continuity we can always translate the encompassing circle some non-zero distance toward the point of tangency such that it comes to touch none of the inner circles, and then by our previous argument shrink the circle radius. This contradicts the assumption that the circle is minimal. 
Lemma 2. The circle of least area encompassing two arbitrary circles is the unique circle drawn tangent to both inner circles such that its diameter connects the two points of tangency.

Proof. By arguments similar to those from Lemma 1, the minimal circle must be tangent to both of the inner circles. Figure 11 shows a general configuration of such a system. Note that $R=r_{1}+l_{1}=r_{2}+l_{2}$ and therefore $R=\left(r_{1}+r_{2}+l_{1}+l_{2}\right) / 2$. For $l_{1}+l_{2} \geq$ $\sqrt{\left(x_{2}-x_{1}\right)^{2}+\left(y_{2}-y_{1}\right)^{2}}, R$ is minimized when $l_{1}+l_{2}$ satisfy with equality. Under this condition the diameter of the circle connects the two points of tangency.

Based on the above two lemmas, we now prove Proposition 1. The smallest circle encompassing three circles cannot be smaller than the smallest circle encompassing a selection of two circles. This is true because the set of circles containing all three is only a subset of the circles containing only two. There are three such selections, so the minimal encompassing circle of all three circles must be as large or larger than the largest circle resulting from a selection. If that encompassing circle contains the third inner circle as well, then it suffices to encompass the system of three. Condition 1 is satisfied.

By Lemma 1 we know that the minimal circle encompassing three must touch at least two of the inner circles. We claim that when Condition 1 is not satisfied Condition 2 must be, for which we must show that a circle touching only two, but not corresponding to the minimal circle encompassing those two, is not a minimal circle encompassing the three. We prove this by contradiction: We assume Condition 1 is not satisfied, but that the minimal encompassing circle touches only two inner circles. That is, we assume the minimal encompassing circle touches two inner circles and contains the third, but does not correspond to a minimal circle encompassing the two circles of tangency. Again, by continuity, we can shift the encompassing circle some non-zero distance such that none of the three circles touch the encompassing circle. Then we can shrink the circle, which contradicts the assumption that the circle is minimal. 


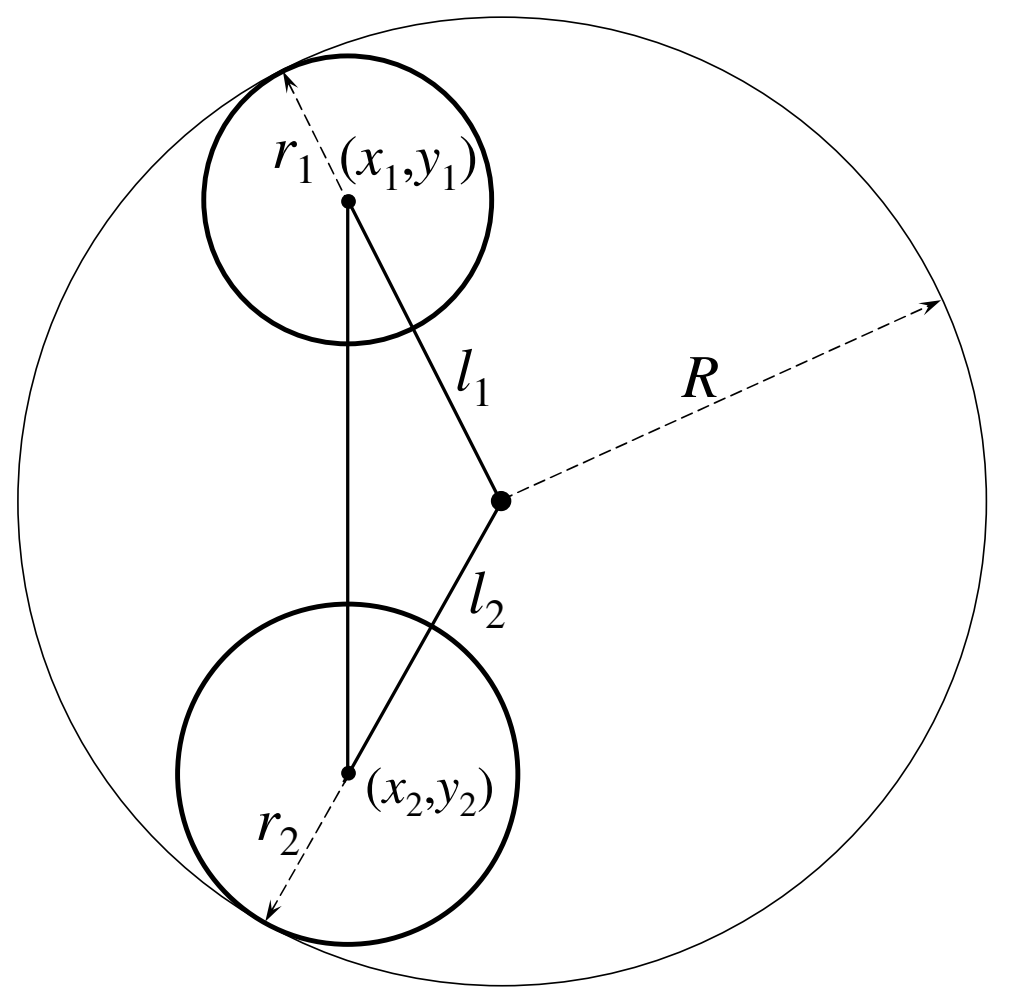

Figure 11: Encompassing circle tangent to two inner circles. 


\section{BIBLIOGRAPHY}

[1] B. Sridhar, K. S. Sheth, and S. Grabbe, "Airspace complexity and its application in air traffic management," in 2nd USA/Europe Air Traffic Management RED Seminar, Orlando, FL, Dec. 1998.

[2] Database, Bureau of Transportation Statistics. [Online]. Available: http://www. transtats.bts.gov/

[3] J. M. Histon, R. J. Hansman, B. Gottlieb, H. Kleinwaks, S. Yenson, D. Delahaye, and S. Puechmorel, "Structural considerations and cognitive complexity in air traffic control," in 19th IEEE/AIAA Digital Avionics Systems Conf., Irvine, CA, Oct. 2002.

[4] A. Yousefi and G. L. Donohue, "Temporal and spatial distribution of airspace complexity for air traffic controller workload-based sectorization," in AIAA 4th Aviation Technology, Integration and Operation Forum, Chicago, IL, Sep. 2004.

[5] M. L. Cummings and C. G. Tsonis, "Deconstructing complexity in air traffic control," in HFES Annual Conference, Orlando, FL, 2005.

[6] U. Metzger and R. Parasuraman, "Automation in future air traffic management: effects of decision aid reliability on controller performance and mental workload," Human Factors, vol. 47, no. 1, pp. 35-49, 2005.

[7] R. W. Simpson, "Course notes for 16.72: Air traffic control," Massachusetts Institute of Technology, Cambridge, MA, 1993.

[8] I. V. Laudeman, S. G. Shelden, R. Branstrom, and C. L. Brasil, "Complexity and automation displays of air traffic control: literature review and analysis," NASA-TM1998-112226, NASA, 1998.

[9] D. Delahaye and S. Puechmorel, "Air traffic complexity: towards intrinsic metrics," in 3rd USA/Europe Air Traffic Management RÉD Seminar, Napoli, Italy, Jun. 2000.

[10] J. Xing and C. A. Manning, "Complexity and automation displays of air traffic control: literature review and analysis," Final Report, DOT/FAA/AM-05/4, 2005. 
[11] V. Sharma, M. Savchenko, E. Frazzoli, and P. G. Voulgaris, "Transfer time complexity of conflict-free vehicle routing with no communications," International Journal of Robotics Research, vol. 26, no. 3, pp. 255-271, Mar. 2007.

[12] R. A. Paielli and H. Erzberger, "Conflict probability estimation for free flight," Journal of Guidance Control and Dynamics, vol. 20, no. 3, pp. 588-596, 1997.

[13] J. M. Hoekstra, R. C. J. Ruigrok, and R. N. H. W. van Gent, "Free flight in a crowded airspace?" Progress in Astronautics and Aeronautics, vol. 193, pp. 533-546, 2001.

[14] J. S. B. Mitchell, V. Polishchuk, and J. Krozel, "Airspace throughput analysis considering stochastic weather," in AIAA Guidance, Navigation, and Control Conference, Keystone, CO, Aug. 2006.

[15] J. Krozel, J. S. B. Mitchell, V. Polishchuk, and J. Prete, "Airspace capacity estimation with convective weather constraints," in AIAA Guidance, Navigation, and Control Conference, Hilton Head, SC, Aug. 2007.

[16] A. M. Bayen, R. L. Raffard, and C. J. Tomlin, "Adjoint-based control of a new Eulerian network model of air traffic flow," IEEE Transactions on Control Systems Technology, vol. 14 , no. 5, pp. 804-818, Sep. 2006.

[17] Z.-H. Mao, E. Feron, and K. Bilimoria, "Stability and performance of intersecting aircraft flows under decentralized conflict avoidance rules," IEEE Transactions on Intelligent Transportation Systems, vol. 2, no. 2, pp. 101-109, Jun. 2001.

[18] Z.-H. Mao and E. Feron, "Stability and performance of intersecting aircraft flows under sequential conflict resolution," in Proc. American Control Conf., vol. 2, Arlington, VA, 2001, pp. 722-729.

[19] Z.-H. Mao, D. Dugail, E. Feron, and K. Bilimoria, "Stability of intersecting aircraft flows using heading change maneuvers for conflict avoidance," IEEE Transactions on Intelligent Transportation Systems, vol. 6, no. 4, pp. 357-369, Dec. 2005.

[20] Z.-H. Mao, D. Dugail, and E. Feron, "Space partition for conflict resolution of intersecting flows of mobile agents," IEEE Transactions on Intelligent Transportation Systems, 2007 (in press).

[21] H. Dörrie, 100 Great Problems of Elementary Mathematics: Their History and Solutions. New York, NY: Dover, 1965. 